\title{
SGT1-Specific Domain Mutations Impair Interactions with the Barley MLA6 Immune Receptor in Association with Loss of NLR Protein
}

\author{
Antony V. E. Chapman, ${ }^{1,2}$ J. Mitch Elmore, ${ }^{2}$ Maxwell McReynolds, ${ }^{2,3}$ Justin W. Walley, ${ }^{1,2,3}$ and \\ Roger P. Wise , $^{1,2,4, \dagger}$ \\ ${ }^{1}$ Interdepartmental Genetics \& Genomics, lowa State University, Ames, IA 50011, U.S.A. \\ ${ }^{2}$ Department of Plant Pathology \& Microbiology, lowa State University, Ames, IA 50011, U.S.A. \\ ${ }^{3}$ Interdepartmental Plant Biology, lowa State University, Ames, IA 50011, U.S.A. \\ ${ }^{4}$ Corn Insects and Crop Genetics Research Unit, USDA-Agricultural Research Service, Ames, IA 50011, U.S.A.
}

Accepted 9 December 2021.

The Mla (Mildew resistance locus a) of barley (Hordeum vulgare $\mathrm{L}$.) is an effective model for cereal immunity against fungal pathogens. Like many resistance proteins, variants of the MLA coiled-coil nucleotide-binding leucine-rich repeat (CCNLR) receptor often require the HRS complex (HSP90, RAR1, and SGT1) to function. However, functional analysis of Sgt1 has been particularly difficult, as deletions are often lethal. Recently, we identified rar3 (required for Mla6 resistance 3), an in-frame $\mathrm{Sgt1}_{\Delta K L 308-309}$ mutation in the SGT1specific domain, that alters resistance conferred by MLA but without lethality. Here, we use autoactive MLA6 and recombinant yeast-two-hybrid strains with stably integrated HvRarl and $\mathrm{HvHsp} 90$ to determine that this mutation weakens but does not entirely disrupt the interaction between SGT1 and

${ }^{\dagger}$ Corresponding author: R. P. Wise; roger.wise@usda.gov

Current address for Antony V. E. Chapman: Phytoform Labs Ltd, Lawes Innovation Hub, West Commons Harpenden, AL5 2JQ, U.K.

Current address for J. Mitch Elmore: USDA-ARS Cereal Disease Laboratory, 1551 Lindig Street, St. Paul, MN 55108, U.S.A.

Mention of trade names or commercial products in this publication is solely for the purpose of providing specific information and does not imply recommendation or endorsement by the USDA or the National Science Foundation. USDA is an equal opportunity provider and employer.

Funding: Research supported in part by National Science Foundation (NSF)-Plant Genome Research Program Division of Integrative Organismal Systems grant 13-39348, United States Department of Agriculture National Institute of Food and Agriculture (USDA-NIFA) grant 2020-67013-31184 and USDA-Agricultural Research Service project 3625-21000-067-00D to R. P. Wise and NSF-IOS 1759023, the Office of Biotechnology, Iowa State University, and USDA NIFA Hatch project IOW3808 to J. W. Walley. The funders had no role in study design, data collection and analysis, decision to publish, or preparation of the manuscript.

*The $\boldsymbol{e}$-Xtra logo stands for "electronic extra" and indicates there are supplementary figures, supplementary tables, and supplementary data published online.

The author(s) declare no conflict of interest. under the Creative Commons CCO "No Rights Reserved" license by waiving all of his or her rights to the work worldwide under copyright law, including all related and neighboring rights, to the extent allowed by law, 2022.
MLA. This causes a concomitant reduction in MLA6 protein accumulation below the apparent threshold required for effective resistance. The $\Delta \mathrm{KL} 308-309$ deletion had a lesser effect on intramolecular interactions than alanine or arginine substitutions, and MLA variants that display diminished interactions with SGT1 appear to be disproportionately affected by the SGT1 $1_{\triangle K L 308-309}$ mutation. We hypothesize that those dimeric plant CC-NLRs that appear unaffected by Sgt1 silencing are those with the strongest intermolecular interactions with it. Combining our data with recent work in CC-NLRs, we propose a cyclical model of the MLA-HRS resistosome interactions.

Keywords: Blumeria graminis f. sp. hordei, chaperone, nucleotidebinding leucine-rich repeat (NLR) receptor, powdery mildew

Fungal pathogens represent a large and economically significant challenge to crops worldwide (Savary et al. 2019). To lessen the impact of fungi on our food sources, we employ a variety of biological, chemical, and mechanical methods, to varying degrees of success. Recent focus has come upon the potential of utilizing molecular genetic methods to quicken the development of genetically resistant crops, which would reduce the need for, and damage caused by, chemical fungicides (Carneiro et al. 2020; Knebel et al. 2019; van Wersch et al. 2020). However, for these methods to be effective and durable, we must understand the molecular interactions between crops and pathogenic fungi as completely as possible (Zhang et al. 2020). Key tools for expanding our understanding are model crop-pathogen systems in which discoveries and innovation can be extrapolated to other crops.

The interaction between barley (Hordeum vulgare L.) and Blumeria graminis $\mathrm{f}$. sp. hordei, the cause of powdery mildew disease, is a model for investigating plant host-fungal pathogen interactions in large-genome cereals (Draz et al. 2019). B. graminis f. sp. hordei is an obligate biotrophic fungus, meaning that it must parasitize living cells to feed (Yamaoka et al. 2006). During infection, fungal appressoria will penetrate the cell wall and release proteins directly into the host cell (Godfrey et al. 2009). These proteins, called effectors, have specialized functions, such as subverting host defense responses and facilitating nutrient absorption (Krattinger and Keller 2016). However, in parallel with the evolution of pathogen effectors, plants have evolved proteins that detect effectors directly or indirectly (Jaswal et al. 2020). Once detected, these resistance $(\mathrm{R})$ proteins initiate severe defense responses that 
often culminate in the death of the infected cell and, consequently, the death of the fungus (Lo Presti et al. 2015).

Alleles of barley mildew resistance locus A (Mla) (Halterman and Wise 2004; Jørgensen 1994; Seeholzer et al. 2010; Wei et al. 2002) encode proteins that provide race-specific defense by recognizing cognate powdery mildew effectors (Bauer et al. 2021; Lu et al. 2016; Ridout et al. 2006; Saur et al. 2019). MLA proteins are archetypical coiled-coil (CC), nucleotide-binding (NB), leucinerich repeat (LRR) receptor proteins, or NLRs (Shirasu 2009; van Wersch et al. 2020) (Fig. 1A). To confer resistance, MLA proteins often require association with the HRS complex, which is composed of cochaperones HSP90 (Heat-Shock Protein 90), RAR1 (Required for Mla12 Resistance 1), and SGT1 (Suppressor of G-two allele of $S k p 1$ ) (Fig. 1B) (Bieri et al. 2004). Once MLA recognizes the presence of the cognate effector, current evidence indicates that the complex translocates to the nucleus, where it initiates a signaling cascade, including interactions with distinct transcription factors, that leads to a hypersensitive reaction and resistance (Bai et al. 2012; Chang et al. 2013; Shen et al. 2007).

The interactions between NLRs and the HRS complex play a critical role in immune response to pathogens (Sun et al. 2020). To identify unknown factors in this pathway, we conducted fastneutron mutagenesis of a resistant barley line, cereal introduction (CI) 16151 (Manchuria background, Mla6), and screened for loss of resistance to $B$. graminis f. sp. hordei isolate $5874\left(A V R_{a 6}\right)$. In the derived susceptible mutant m11526, we identified a 6-bp, in-frame Lys-Leu deletion within the SGT1-specific (SGS) domain of HvSgtl (HORVU3H1G055920) as causing selective disruption of Mla-mediated, race-specific resistance to powdery mildew (Chapman et al. 2020). The required for mla6 resistance (rar3) mutation $S_{g t 1_{\Delta K L 308-309}}$ alters the function of Mla6, Mla7, and Mla12 (designated Rar3-dependent alleles) but did not appear

A

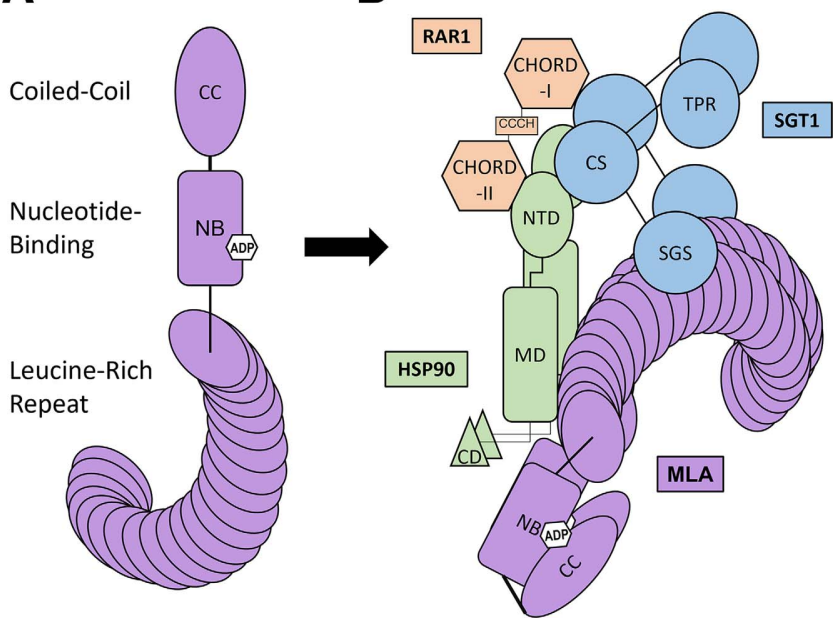

Fig. 1. Model for the structure of MLA and how it interacts with the HRS complex composed of HSP90 (Heat-Shock Protein 90), RAR1 (Required for Mla12 Resistance 1), and SGT1 (Suppressor of G-two allele of Skp1), adapted from (Boter et al. 2007; Shirasu 2009; Siligardi et al. 2018). A, The MLA protein is comprised of the coiled-coil (CC) signaling, nucleotidebinding (NB), and leucine-rich repeat (LRR) domains. The MLA NB domain binds adenosine di-phosphate (ADP) in its inactive state, which maintains intramolecular interactions with the $\mathrm{CC}$ domain to prevent signaling. B, MLA is required to interact with the HRS complex to function, this consists of two units of HSP90, one unit of RAR1, and two units of SGT1. RAR1 consists of the cysteine- and histidine-rich domains (CHORD-I and CHORD-II) and the cysteine- and histidine-containing motif $(\mathrm{CCCH})$. HSP90 consists of the N-terminal (NTD), middle (MD), and C-terminal dimerization (CTD) domains. SGT1 consists of the TPR (tetratricopeptide repeat), CS (CHORD-SGT1), and SGS (SGT1-specific) domains. to affect the function of Mla1, Mla9, Mla10, and Mla13 (designated Rar3-independent alleles).

Sgtl is critical for a vast array of eukaryotic cell processes, as evidenced by the lethality of deletion mutants (Azevedo et al. 2002; Martins et al. 2009), or when it is silenced (Bhattarai et al. 2007; Thao et al. 2007), whereas the rar3-m11526 mutant is morphologically identical to the CI 16151 progenitor from seed to seed (Chapman et al. 2020). This suggests that the $S g t 1_{\Delta K L 308-309}$ mutation explicitly inhibits $M l a$-specified disease resistance but does not overtly affect other cell processes that Sgtl is involved with, such as hormone detection and kinetochore development (Meldau et al. 2011). Thus, the $S g t 1_{\Delta K L 308-309}$ mutation provides a unique opportunity to investigate the involvement of Sgt1 in immunity.

To explore the intermolecular and structural relationship between SGT1 and NLRs, we took advantage of $S g t 1_{\triangle K L 308-309}$ to investigate how it perturbs MLA-dependent disease resistance. First, we show that the previously unobserved interaction between MLA6 and SGT1 can be revealed in the yeast-two-hybrid (Y2H) protein-protein interaction system (Dreze et al. 2010), by utilizing full-length autoactive NLR mutants and reconstituting the HRS complex through yeast genome engineering. Using this system, we then show that SGT1 $1_{\Delta \mathrm{KL} 308-309}$ destabilizes the interaction with both the Rar3-independent MLA1 and Rar3-dependent MLA6, as compared with wild-type (WT) SGT1. Lastly, parallel reaction monitoring (PRM)-based targeted mass spectrometry (MS) showed that MLA6 protein accumulates in the $\operatorname{Sgtl}_{\triangle K L 308-309}$ mutant line to a lower, but still observable, level than in WT CI 16151 in both unchallenged plants and plants inoculated with powdery mildew. Combined with other recent evidence, these results suggest that SGT1 may be required by more NLRs than previously suspected. Moreover, the $\mathrm{Sgtl}_{\triangle K L 308-309}$ mutation may be a new tool for investigating NLRs by separating disease resistance signaling from other cellular processes.

\section{RESULTS}

Incorporating barley HSP90 and RAR1 into Y8800
yeast facilitates interactions between SGT1 and MLA

We previously demonstrated that the rar3 mutation localized to an in-frame Lys-Leu deletion within the SGS domain of SGT1 (Chapman et al. 2020). The LRR domain of NLRs interacts directly with the SGS domain of SGT1 (Fig. 1) (Bieri et al. 2004). Thus, the Lys-Leu deletion may be impairing the interaction between NLRs and SGT1, resulting in an altered immune response. SGT1 functions as part of the HRS complex, along with HSP90 and RAR1, and it has been shown that MLA6 requires both HSP90 and RAR1 to function (Bieri et al. 2004). Further, it has been suggested that RAR1 may increase the affinity of SGT1 for NLRs (Kadota and Shirasu 2012). Therefore, it is possible that the lack of barley HSP90 and RAR1 in the Y2H system prevents some NLRs, such as MLA6, from interacting properly with SGT1. We hypothesized that reconstituting the barley HRS complex in the $\mathrm{Y} 2 \mathrm{H}$ system could be used to test interactions between SGT1 and NLRs.

First, we compared the interaction between MLA and WT SGT1 or the SGT1 $1_{\triangle \mathrm{KL} 308-309}$ mutant (GenBank MT787218) using the GAL4 Y2H system (Dreze et al. 2010). Previously, it had been required to use only the LRR domain of NLRs to test for these interactions, as the whole NLR is autoinhibited (Bieri et al. 2004). This could be due to the NLRs existing in a closed conformation naturally until they are activated by a cognate effector or brought into the HRS scaffolding (Bernoux et al. 2016). We tested multiple fragments of the MLA1 LRR, which had been shown previously to interact with SGT1 (Bieri et al. 2004), against SGT1 WT and $\mathrm{SGT}_{\Delta \mathrm{KL} 308-309}$ (Fig. 2). We found that MLA LRR fragment 543-958 gave the strongest interaction 
(Fig. 2B2) with SGT1 WT (as determined by the rate and size of yeast colony growth), and that the interaction was slightly, but consistently, weaker with SGT1 ${ }_{\Delta \mathrm{KL} 308-309}($ Fig. 2B3). However, consistent with previous reports, we did not observe an interaction between SGT1 and either full-length MLA6 or the equivalent LRR fragment, indicated by the observed growth not being significantly different than the negative controls (Fig. 2E2 and F2).

Then, to test whether the inclusion of cochaperones would facilitate the interaction between MLA6 and SGT1, HvRarl and $\mathrm{HvHsp} 90$ were stably integrated into the genome of Y8800 yeast to generate the "Y-archer" strain Y-RcHa (Y8800-Rarl-cyclHsp90-adh1) (Mikkelsen et al. 2012). When the interaction tests between SGT1 and MLA fragments were repeated using the Y-RcHa strain instead of Y8800, we saw increased evidence of interaction between MLA1 and SGT1 (Fig. 2B4). However, similar to the tests performed with the original Y8800 strain, an increased interaction using Y-RcHa was not observed in the interaction between MLA1 and SGT1 ${ }_{\Delta \mathrm{KL} 308-309}$ (Fig. 2B5), and there was still no observed interaction between MLA6 and SGT1 (Fig. 2E4 and F4). As Mlal was found to be a Rar3-independent allele, this suggested that the $S_{S t} 1_{\Delta K L 308-309}$ mutation disrupts interactions with certain MLA variants but not others (Chapman et al. 2020)

\section{Site-directed mutagenesis of Sgt1 suggests KL308-309} is not a direct interaction site between SGT1 and MLA1.

To investigate further into why the $\mathrm{SGT}_{\Delta \mathrm{KL} 308-309}$ deletion affects interactions, we generated a series of Sgtl mutant constructs that would delineate the possible molecular mechanisms behind the observed disruption. Using site-directed mutagenesis, we generated constructs with only one of the two candidate amino acids deleted, to determine if a single position was causative (Fig. 3, $S g t 1_{\Delta K 308}$ and $S g t 1_{\Delta L 309}$ ). In addition, we generated two single and one double alanine substitutions to determine whether the WT residue side chains were necessary for function or if it was the loss of the position that caused the phenotype regardless of the side chain (Fig. 3, Sgt1 ${ }_{K 308 A}, S g t 1_{L 309 A}$, and $\left.S g t 1_{K L 308 A A}\right)$. Lastly, as lysine is known to form intermolecular salt bridges with aspartates or glutamates (Kumar and Nussinov 2002) and we were looking for the cause behind a weakened interaction, we generated $\operatorname{Sgt}_{K 308 R}$ (Fig. 3). The arginine in this construct would conserve the side chain nitrogen atoms required for the salt bridge to form, if that is the function of $\mathrm{SGT}_{\mathrm{K} 308}$.
Interestingly, it was $\mathrm{SGT}_{\Delta \mathrm{L} 309}$ that appeared to mimic the lesser interaction of $\mathrm{SGT}_{\triangle \mathrm{KL} 308-309}$ with MLA, though the differences between the two interactions is small (Fig. 3B5). In contrast, neither of the single alanine substitutions appeared to have weakened interactions similar to the original $\mathrm{Sgt1}_{\triangle K L 308-309}$ (Fig. 3B6 and B7). However, the double alanine and the arginine substitutions both showed a complete loss of interaction (Fig. 3B8 and B9).

As alanine is a relatively unreactive amino acid, it is surprising that the double alanine substitution had a greater effect on the strength of the interaction than the double deletion. The effect of the $S g t 1_{K 308 R}$ mutation may suggest that the function of $\mathrm{SGT}_{\mathrm{K} 308}$ is not in forming intermolecular salt bridges. However, it has been shown before that mutating lysine to arginine can still disrupt established salt bridges (Joseph et al. 2018). In addition, the two amino acids after the deletion, which could be considered as taking the place of the deleted residues, are aspartate (D) and glycine (G). As aspartate forms the opposite side of a salt bridge to lysine, an aspartate replacing a lysine should destroy the proposed salt bridge. Alas, the deletion only causes a slight weakening of the interaction, especially when compared with alanine substitutions, also supporting the possibility that $\mathrm{SGT}_{\mathrm{K} 308}$ is not involved in intermolecular salt-bridge formation. In this regard, arginine has a guanidinium group that allows for more possibilities for interactions because of the additional nitrogen atoms and it has been shown to increase intramolecular stability when compared with lysine (Sokalingam et al. 2012). Therefore, as the SGS domain is considered extremely disordered due to its lack of a defined tertiary structure (Taube et al. 2014), the $S g t 1_{K 308 R}$ mutation may cause an increase in stability of the SGS domain that somehow impairs its function.

\section{MLA $_{797}$ and MLA $_{798}$ are essential for interactions with SGT1.}

Previously, it was found that the requirement for Rarl by Mla6 and Mla13 could be delineated to a single position in the LRR region, MLA $_{721}$ (Halterman and Wise 2004). However, MLA6 and RAR1 do not directly interact (Bieri et al. 2004). Rather, RAR1 interacts with both SGT1 and HSP90 and could function as structural support for the HRS complex to maintain it in a conformation that promotes interaction with $\mathrm{R}$ proteins (Boter et al. 2007). Therefore, we hypothesize that $\mathrm{MLA}_{721}$ confers requirement for Rarl by affecting the interaction between MLA and SGT1. It is interesting that Rarl-independent NLRs,

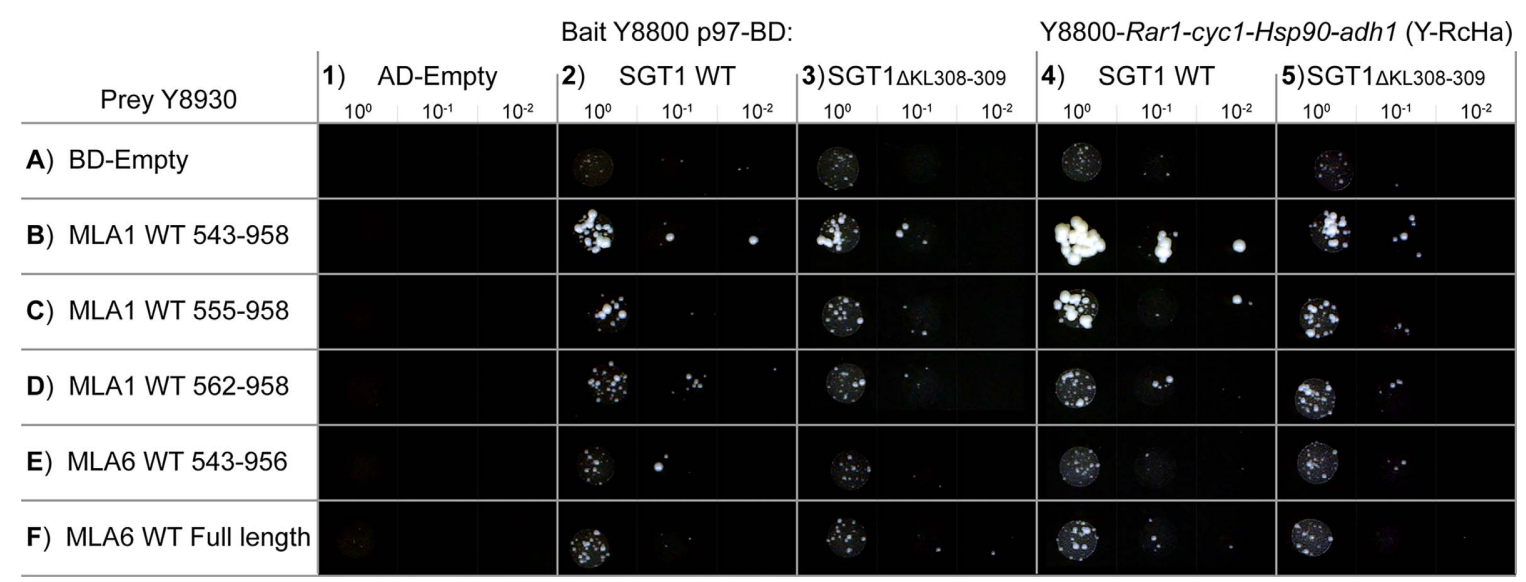

Fig. 2. Yeast-two-hybrid interaction tests between MLA1 or MLA6 fragments and SGT1 1 KL308-309 with the original Y8800 strain or modified Y-RcHa strain. Rows and columns are identified by letters and numbers, respectively. For example, AD-Empty/BD-Empty test is referenced as 2A1. Diploid Y8800 or Y-RcHa/Y8930 yeast expressing activation or binding domains (AD and BD, respectively) were grown on media lacking histidine (SC-LWH). MLA1 WT 543-958 indicates that the fragment of MLA1 is from the wild-type sequence between amino acid position 543 to the stop codon at 958. MLA6 is two amino acids shorter than MLA1, therefore 956 corresponds to the MLA6 stop codon. Yeast was grown on SC-LWH, and images were taken 4 days after plating. Dilutions of diploids cells for plating are $10^{0}, 10^{-1}$, and $10^{-2}$ of the original 0.5 optical density culture. 
such as MLA1, have an aspartate at this location, as they are capable of forming intermolecular salt bridges, whereas the glycine that Rarl-dependent NLRs, such as MLA6, have at this position are inactive. Therefore, $M L A 1_{\mathrm{D} 721}$ may form an intermolecular salt bridge with SGT1. However, if this is the case, it may not be the only site of interaction between the two proteins, as this change is not enough to confer a MLA1-equivalent resistance response (Halterman and Wise 2004). Therefore, we extrapolate that there may be a position within MLA that confers requirement for $S g t l_{K L 308-309}$.

To investigate key sites of interaction between MLA and SGT1, we scrutinized the differential "Rar3" requirement by MLA variants for amino acid consistencies between those defined as Rar3-dependent or -independent (Chapman et al. 2020) (Fig. 4). We identified three major sites that appear to show conservation or redundancy within each group. At $\mathrm{MLA}_{708}$, Rar3dependent variants have glutamate $(\mathrm{E})$, whereas the Rar3-independent variants have either asparagine (N) and aspartate (D). These three amino acids are very similar and, therefore, are generally interchangeable. They are polar, which means they prefer to be on the surface of proteins, exposed to an aqueous environment. Glutamate and aspartate are more similar in function than asparagine and aspartate. However, there are some key differences; while glutamate and aspartate can both form intermolecular salt bridges, asparagine does not, which would suggest that if the N/D at this position confers a stronger interaction with SGT1, it would not be due to the gaining of a salt bridge. The main similarity between asparagine and aspartate, which is not shared by glutamate, is the slightly shorter side chain, which may suggest that its function may be structural, if this is the key difference.

At MLA ${ }_{797}$, MLA6 has a uniquely deleted amino acid, whereas the other Rar3-dependent variants have tryptophan (W) and the Rar3-independent variants have either serine (S) or threonine (T). It is hard to attribute a function to a missing amino acid; however, it may be that, in this case, tryptophan is not performing any particular function or it is negatively associated with interactions and, therefore, a deletion could be functionally preferable. Conversely, serine and threonine are both small,
AA Position (Relative to MLA1)

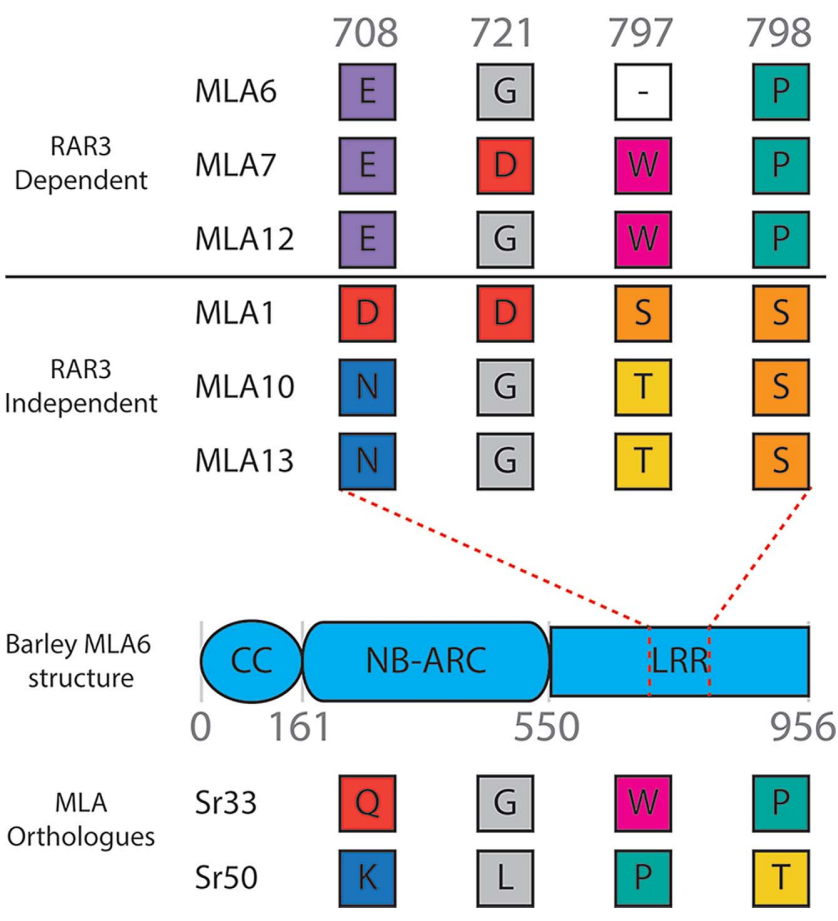

Fig. 4. Conservation of amino acids between Rar3-dependent or -independent MLA variants at multiple key sites. Conservation is observed between Rar3-dependent alleles at 708 and between Rar3-independent alleles at 798. At position 797, Rar3-independent alleles contain either serine or threonine, which can be functionally redundant. Position 721, which confers genetic requirement for Rarl, does not show conservation between the groups (Halterman and Wise 2004). Below the structure are two MLA orthologs in wheat, Sr33 and Sr50 (Chen et al. 2017; Periyannan et al. 2013). Amino acid positions are labeled according to an amino acid alignment of all six alleles relative to MLA1. $\mathrm{E}=$ glutamate, $\mathrm{D}=$ aspartate, $\mathrm{N}=$ asparagine, $\mathrm{G}=$ glycine, $\mathrm{W}=$ tryptophan, $\mathrm{S}=$ serine, $\mathrm{T}=$ threonine, $\mathrm{P}=$ proline, $\mathrm{K}=$ lysine, $\mathrm{L}=$ leucine, $\mathrm{Q}=$ glutamine, and "-" designates a gap in the alignment.

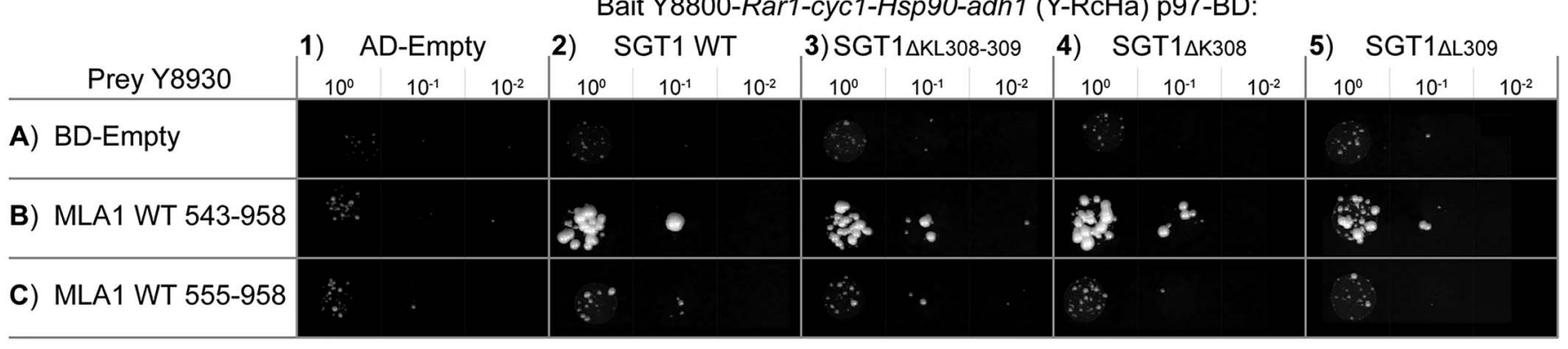

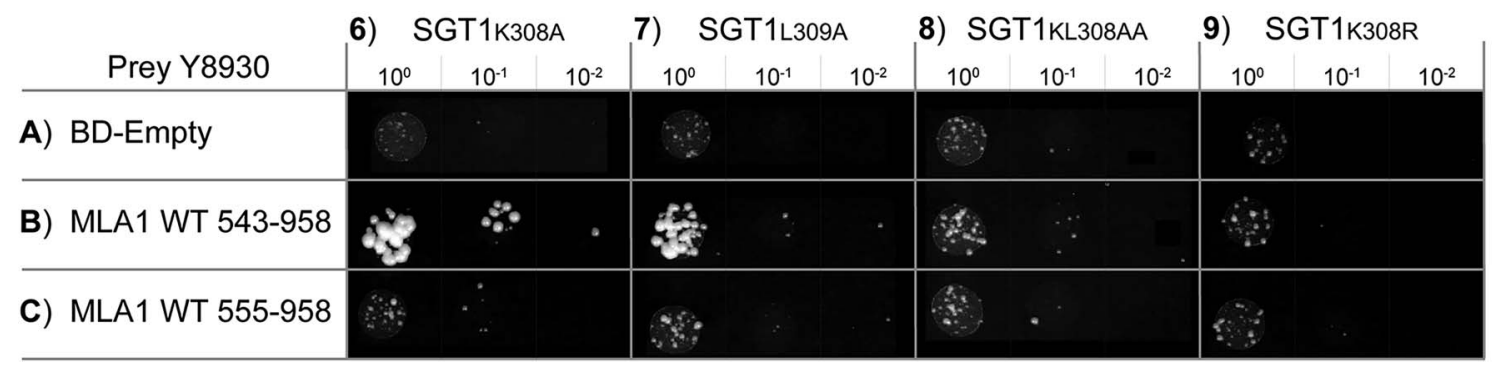

Fig. 3. Yeast-two-hybrid interaction tests between MLA1 leucine-rich repeat fragments and SGT1 mutants. Rows and columns are identified by letters and numbers, respectively. For example, AD-Empty/BD-Empty negative control is 3A1. Diploid Y8800 or Y-RcHa/Y8930 yeast expressing activation or binding domains (AD and BD, respectively) were grown on media lacking histidine (SC-LWH). MLA1 WT 543-958 indicates that the fragment of MLA1 is from the wild-type sequence between amino acid position 543 to the stop codon at 958 . Yeast was grown on SC-LWH, and images were taken 4 days after plating. Dilutions of diploids cells for plating are $10^{0}, 10^{-1}$, and $10^{-2}$ of the original 0.5 optical density culture. 
polar, and can be phosphorylated. The phosphorylation of the LRR domains of proteins has not been observed in NLRs but has been observed in many receptor kinases and receptor-like kinases (Klymiuk et al. 2021; Park et al. 2012).

Position $\mathrm{MLA}_{798}$ has the starkest contrast between the two groups. Rar3-dependent variants have a proline $(\mathrm{P})$, whereas the Rar3-independent variants have serine (S). However, it is possible for the serine side chain to form a hydrogen bond with the protein background whereby it acts as proline does structurally. Additionally, proline is fairly nonreactive. Therefore, it is possible that at this position, proline and serine act similarly structurally, while the serine in Rar3-independent variants performs an additional function that confers a stronger interaction with SGT1.

When we tested MLA1 and MLA6 site-directed mutants for interaction with SGT1, we observed that mutating MLA1 $1_{797}$ and MLA1 $_{798}$ to the Rar3-dependent forms $\left(\right.$ MLA1 $_{\Delta 7975}$ and MLA1 $1_{\text {S798P }}$ ) completely disrupted the interaction between MLA1 and SGT1 (Fig. 5E2 and F2). In contrast, the reciprocal mutations MLA6 $_{+7975}$ and MLA6 ${ }_{\text {P797S }}$ did not cause an equivalent gain of interaction (Fig. 5H2 and I2). However, we did observe an increase in interaction between MLA6 $6_{+7975}$ and SGT1 WT, whereas the interaction between MLA6 $6_{+797 S}$ and SGT1 ${ }_{\Delta \mathrm{KL} 308-309}$ was weaker (Fig. 5I3). This is similar to the findings shown by Halterman and Wise (2004) whereby the $\mathrm{MLA6}_{\mathrm{G} 721 \mathrm{D}}$ mutation alleviated the functional requirement for RAR1, but the reciprocal mutant MLA1 $1_{\mathrm{D} 721 \mathrm{G}}$ did not gain a requirement for RAR1. Mutating $\mathrm{MLA1}_{708}$ did not appear to affect interaction strength (Fig. 5D2). The proximity of the two positions that appear important for MLA1 to interact with SGT1, MLA1 ${ }_{797}$ and MLA1 $_{798}$, may suggest an additive function whereby both are required to confer a strong interaction with SGT1 and, consequently, "Rar3-independence".

One of the theorized dynamics between MLA and the HRS complex is that the NLR naturally exists in an inactive, closed conformation (Ade et al. 2007; Wang et al. 2015b, 2019b). If the R protein must be activated before it can interact with the HRS complex, that would explain why we don't observe an interaction between full length MLA1 and SGT1 but instead must use only the LRR domain. To test the theory that full length MLA needs to be activated before it interacts with the HRS complex, we generated an autoactive version of full length MLA6, MLA6 $6_{\mathrm{D} 502 \mathrm{~V}}$, which has a mutation in the MHD motif of the NB domain that mimics ATP binding (Tameling et al. 2006). We found a small interaction when we tested MLA6 $6_{\mathrm{D} 502 \mathrm{~V}}$ against SGT1 WT but not with $\mathrm{SGT}_{\Delta \mathrm{KL} 308-309}$ (Fig. 5K2 and K3). This suggests that locking MLA6 in an active conformation and reconstituting the HRS complex facilitates the interaction between MLA6 and SGT1 to a level that can be detected using the Y2H Y-archer assay.

\section{Silencing of $\mathrm{NbSgt1}$ prevents cell death caused by autoactive $\mathrm{Mla}_{\mathrm{D5O2V}}$ and $\mathrm{Mla} 7_{\mathrm{D502V}}$.}

Plants with the rar3 $\mathrm{SGT}_{\Delta \mathrm{KL} 308-309}$ mutation disrupt Mlamediated accumulation of $\mathrm{H}_{2} \mathrm{O}_{2}$ and hypersensitive cell death (HR) (Chapman et al. 2020). To further investigate how the SGT1 $_{\Delta \text { KL308-309 }}$ mutation and MLA interact in planta, we utilized the heterologous Nicotiana benthamiana system. To activate the HR in this system, we made use of autoactive Mla constructs $M l a 6_{D 502 V}$ and $M l a 7_{D 502 V}$, whereby D > V mutations in the MHD motif in the NB domain prevent hydrolysis of ATP and cause the MLA protein to be permanently stuck in the "On" conformation (Tameling et al. 2006). Infiltration of these two constructs effectively induced cell death. However, if we preinfiltrated with RNAi:NbSgt1 in an overlapping region, cell death was abolished (Fig. 6; Supplementary Fig. S1).

Then, to test how the SGT1 ${ }_{\triangle K L} 308-309$ mutation and MLA interact, we overexpressed $35 \mathrm{~s}: H v S g t 1$ or $35 \mathrm{~s}: H v S g t 1_{\triangle K L 308-309}$. Although Arabidopsis Sgtl has been shown to complement silenced NbSgtl (Boter et al. 2007), it has recently been suggested that SA signaling brought about by silencing $S g t 1$ in $N$. benthamiana may inhibit subsequent transient expression of genes by Agrobacterium strains ( $\mathrm{Yu}$ et al. 2019). To overcome this, we attempted to express $\mathrm{HvSgtl}$ prior to silencing $\mathrm{NbSgt1}$, hypothesizing that $\mathrm{HvSgtl}$ would be able to complement $\mathrm{NbSgtl}$ and allow subsequent expression of transgenes.

We infiltrated the 35s:HvSgtl or 35s:HvSgtl ${ }_{\triangle K L 308-309}$ (Fig. 6, red-bordered central region) at 2 to 4 days before infiltration with the RNAi:NbSgtl construct in the same region. Then, 2 days later, the cell-death construct was infiltrated, overlapping the previously infiltrated region (Fig. 6, black-bordered overlapping region). Although, silencing NbSgtl consistently prevented cell death from both $M_{1 a 6_{D 502 V}}$ and Mla $7_{D 502 V}$, complementation of NbSgtl silencing was not observed when either 35s:HvSgtl or 35s:HvSgt1 ${ }_{\Delta K L 308-309}$ was infiltrated.

Isolation and sequencing of the HvSgtl constructs from the Agrobacterium strains that were used confirmed that the constructs do not have any errors that may prevent expression or function. The $35 \mathrm{~S}$ overexpression construct pEG100 was used for both $\mathrm{HvSgtl}$ and Mla; therefore, it is unlikely that the construct itself is the issue, as the Mla cell-death construct appeared to express well, indicated by the intense cell-death phenotypes.

\section{MLA6 protein abundance is reduced by the $\operatorname{Sgt1}_{\Delta K L 308-309}$ mutation.}

RAR1 and SGT1 are implicated in maintaining R-protein abundance, and we have shown that the $S g t 1_{\Delta K L 308-309}$ mutation appears to destabilize the interaction between SGT1 and MLA6. Therefore, we could expect the abundance of MLA6 to be significantly lower in the $S g t 1_{\triangle K L 308-309}$ mutant line m11526 as compared with the WT progenitor CI 16151. Previously, it was observed that, despite similar transcript levels of hemagglutinintagged transgenes, MLA1 accumulates to almost four times the levels of MLA6 (Bieri et al. 2004). However, both proteins exhibit a reduction of accumulation in rarl lines, despite a visible disease phenotype only appearing in lines also harboring Mla6. Interaction with SGT1 is necessary for the accumulation of many R proteins (Azevedo et al. 2006; Boter et al. 2007; Mestre and Baulcombe 2006); therefore, the reason MLA1 functions in rarl backgrounds could be due to its inherently stronger interaction with SGT1 and therefore a higher stability that maintains MLA1 above a certain threshold required for HR activation. Thus, the differing effect of $S g t 1_{\Delta K L 308-309}$ on MLA variants may be due to a similar action as lacking RAR1.

To test this hypothesis, we utilized a targeted MS assay termed PRM to quantify the abundance of endogenous MLA6 in the Sgt1 $_{\triangle K L 308-309}$ mutant rar3-m11526 (Bourmaud et al. 2016; Rauniyar 2015). PRM involves targeting multiple predefined precursor ions simultaneously by mass to charge ratio $(\mathrm{m} / \mathrm{z})$ before fragmentation and ion measurement. The specificity of the $\mathrm{m} / \mathrm{z}$ allows for less noise and higher sensitivity than other methods, such as selected reaction monitoring (Peterson et al. 2012). To measure protein abundances in barley samples, we designed and synthesized isotopically heavy versions of peptide candidates, which serve as reference peptides and enable accurate relative quantification between samples. Three MLA and two SGT1 peptide candidates were selected and synthesized (ThermoFisher, Waltham, MA, U.S.A.) (Table 1). HvSGT1_pep1 had been previously detected in maize (Méchin et al. 2007), which made it a promising candidate, whereas neither HvSGT1_pep2 nor any of the MLA peptides were identified previously. Of the MLA peptides, MLA6.9.13_pep2 and MLA6.7.12_pep3 were designed for targeting multiple alleles of MLA. MLA6.9.13_ pep2 is unique to MLA but is present in the MLA alleles MLA6, MLA9, and MLA13, whereas MLA6.7.12_pep3 targets MLA6, MLA7, and MLA12. The manufacturer also supplied a standard peptide, ATLAS, which is not present in the barley proteome. 
To test the quality and determine their fragment ion profile, we injected each synthetic peptide into the MS, using our PRM acquisition settings at varying amounts $(1,100$, and 1,000 fm). From this survey, we could reliably detect the peptides ATLAS, MLA6.7.12_pep3 (hereafter designated MLA6 WaVe), and HvSGT1_pep2 (hereafter designated SGT1 DANK) at both 100 and $1,000 \mathrm{fm}$ amounts. Once candidate peptides were chosen, complex CI 16151 samples were tested with $100 \mathrm{fm}$ of MLA6 WaVe and SGT1 DANK synthetic peptides spiked in. Endogenous peptides were detected for both SGT1 DANK and MLA6 WaVe, eluting at about $55.5 \mathrm{~min}$ and $77.5 \mathrm{~min}$, respectively (Fig. 7). Therefore, we deemed our method suitable for quantification of MLA peptides in complex barley samples. For subsequent runs, the amount of synthetic peptide spiked in was adjusted so that their relative intensities were closer to the endogenous. We used $200 \mathrm{fm}$ for SGT1 DANK and $10 \mathrm{fm}$ for MLA6 WaVe, as $10 \mathrm{fm}$ seemed to be the lower boundary for confidence in detection of the heavy synthetic peptide.

To test whether the $\operatorname{Sgt1}_{\triangle K L 308-309}$ mutation reduced MLA6 abundance, we ran PRM on complex peptide samples isolated from CI 16151 (Mla6, Sgtl, Rarl), m18982 (mla6, Sgt1, Rarl), and m11526 (Mla6, Sgt1 ${ }_{\Delta K L 308-309}$, Rarl) with heavy synthetic SGT1 DANK and MLA6 WaVe spiked in. To compare, we also included Sultan-5 (Mla12, Sgt1, Rarl) and m100 (Mla12, Sgt1, rarl), as the Sultan-5 derived, rarl mutation had been shown previously to reduce MLA accumulation (Bieri et al. 2004). MLA6 WaVe is identical to the corresponding MLA12 peptide, so it can be used for relative quantitation in both the CI 16151 and Sultan-5 backgrounds. The results were exported into
Skyline software, where the appropriate peaks were identified, and peak boundaries were selected and were then quantified by computing the peak area.

When we examine the ratio of light/heavy MLA6 WaVe (Fig. 8), we observe that the mla6 deletion mutant m18982 and the rar3 $S_{g t 1_{\triangle K L 308-309}}$ mutant $\mathrm{m} 11526$ both have significantly reduced levels of detected MLA6. In fact, m11526 has nearly half as much MLA6 abundance as CI 16151, whereas m18982 had a minor amount detected, though we predict there should be none. For confidence in the isolation of the target MLA6 peptide, we require observation of all ions previously characterized for its profile, $\gamma 4, \gamma 5$, and $\gamma 6$ (Fig. 7). However, when we examine the chromatogram for the most abundant m18982 replicate (Supplementary Fig. S2), we observe that only a single ion is observed, i.e., $\gamma 5$. Additionally, if the abundance of MLA6 in m18982 was just below the lower boundary of detection, we would expect to observe the highest intensity ion first, which would be $\gamma 6$, not $\gamma 5$. Therefore, we believe it would be fair to argue that no MLA6 is detected in the mla6 mutant, as would be expected from a deletion mutant. Examining the actual peak area for each biological replication, we observe consistency between the amount of heavy MLA6 WaVe detected, giving confidence to the ratio differences not being an artifact of varying synthetic amounts (Supplementary Fig. S3).

We observed that the rarl mutant $\mathrm{m} 100$ had significantly lower MLA WaVe abundance than its WT progenitor Sultan-5, which is consistent with previous data (Bieri et al. 2004). Interestingly, we also observed that both Sultan-5 and rarl-m100 had significantly higher abundances of MLA WaVe than CI

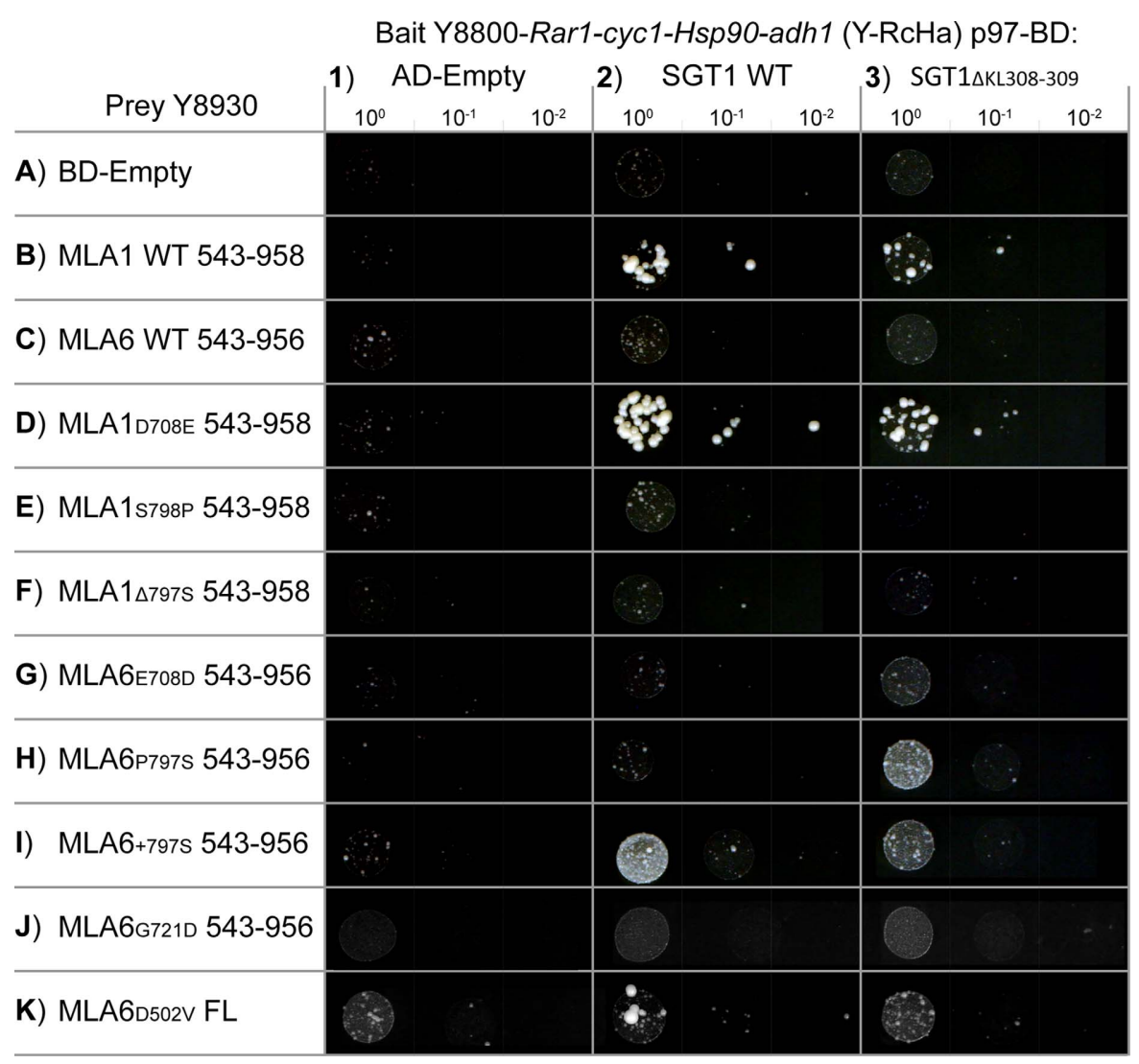

Fig. 5. Yeast-two-hybrid interaction tests between MLA1 or MLA6 mutant leucine-rich repeat fragments and SGT1. Rows and columns are identified by letters and numbers respectively. For example, AD-Empty/BD-Empty test is 5A1. Diploid Y8800 or Y-RcHa/Y8930 yeast expressing activation or binding domains (AD and BD, respectively) were grown on media lacking histidine (SC-LWH). "MLA1 WT 543-958" indicates that the fragment of MLA1 is from the wild-type sequence between amino acid position 543 to the stop codon at 958. MLA6 is two amino acids shorter than MLA1, therefore 956 corresponds to the MLA6 stop codon. Yeast was grown on SC-LWH, and images were taken 4 days after plating. Dilutions of diploid cells for plating are $10^{0}, 10^{-1}$, and $10^{-2}$ of the original 0.5 optical density culture. 
16151 (Supplementary Fig. S4A). This may be due to the differences in genetic background between CI 16151 (Manchuria background) and Sultan-5. In contrast to MLA6, SGT1 does not appear to vary in its abundance between lines as there were no significant differences in the ratios observed in any genotype (Fig. 8; Supplementary Fig. S4B).

Previously, we showed that Mla6 and Sgt1 transcript levels increase markedly during penetration and are significantly different between WT CI 16151 and derived susceptible mutants (Chapman et al. 2020). Thus, we tested the effect of powdery mildew challenge on accumulation of SGT1 and MLA6 proteins. We performed a randomized block experiment in which we inoculated three replicates of CI 16151 (Mla6, Sgt1, Rarl), m18982 (mla6, Sgt1, Rar1), and m11526 (Mla6, sgt1 ${ }_{\Delta K L 308-309,}$ Rarl) at the same growth stage as the above test (Fig. 8) with B. graminis f. sp. hordei $5874\left(A V R_{a 6}\right)$. We sampled first leaves at $16 \mathrm{~h}$ after inoculation (HAI), corresponding to peak accumulation of Mla6 transcripts (Chapman et al. 2020). We performed sample preparation and PRM using the same method as the

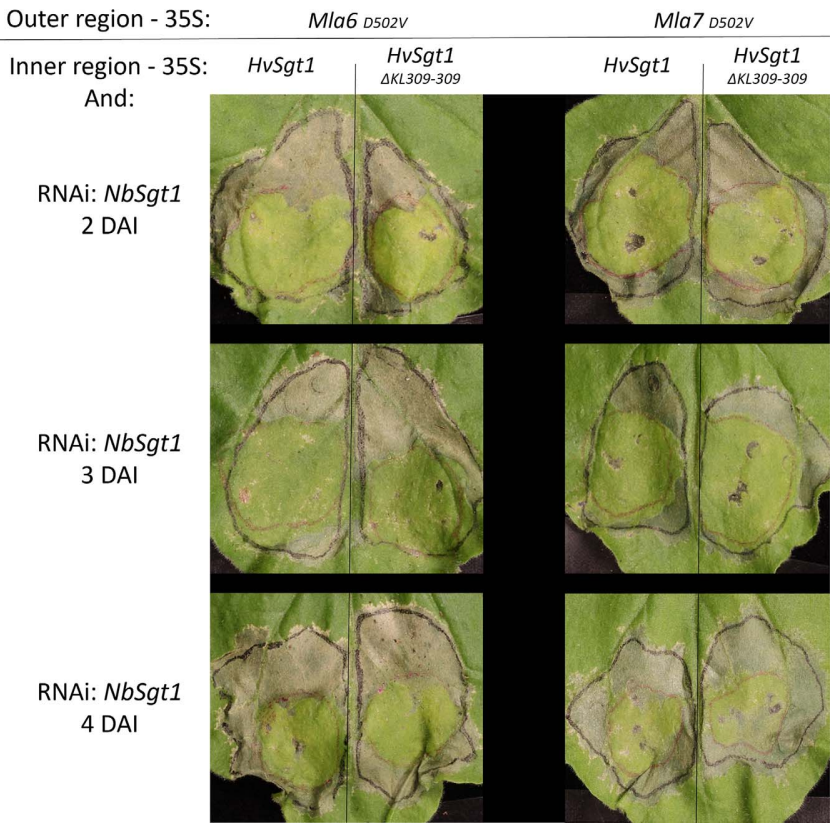

Fig. 6. Transient silencing of NbSgt1 overlapped with overexpression of MLA cell-death constructs. In chronological order, the constructs expressing 35S:HvSgt1 or $35 \mathrm{~S}: H v S g t 1_{\triangle K L 308-309}$ were infiltrated into the center of red-bordered central regions. At 2 to 4 days after infiltration (DAI) of the $H v S g t 1$ constructs, RNAi:NbSgtl constructs were infiltrated into the same red-bordered central regions, and 2 days later, the fulllength $35 \mathrm{~S}$ : $M l a 6_{D 502 \mathrm{~V}}$ or $35 \mathrm{~S}$ : Mla $7_{D 502 \mathrm{~V}}$ cell-death constructs were infiltrated (black-bordered out region) overlapping the red-bordered central region. Leaves were taken, phenotyped, and photographed 2 days after the final infiltration. All infiltrations were performed with Agrobacterium sp. strain EHA105. Additional infiltrations showing nonoverlapping infiltrations are shown in Supplementary Figure S1. uninfected samples to examine SGT1 and MLA6 abundance in the infected tissue and compared the results.

Comparing the relative abundance of MLA6 and SGT1 during infection at $16 \mathrm{HAI}$ to noninoculated samples, we observed no significant change in the abundance of either protein in either mutant (Fig. 9). This suggests that, despite the change in transcript levels, complex posttranscriptional processes maintain protein levels set by the associated genotypes.

\section{DISCUSSION}

\section{RAR1 and HSP90 facilitate interactions between SGT1 and MLA in Y2H systems.}

To investigate domains involved in SGT1-NLR interactions, we used yeast genome engineering to modify the contextual protein environment in the $\mathrm{Y} 2 \mathrm{H}$ system to be closer aligned to in-planta barley. Previously, it was shown in yeast that MLA6 NLR does not interact with SGT1 whereas MLA1 does, even though Mla6 function is disrupted by Sgtl silencing and Mlal is not (Azevedo et al. 2006; Bieri et al. 2004). However, it was also shown that Mla6 requires Rarl and $H s p 90$ in planta to function and the interaction itself may be ephemeral or dependent on prior activation by the cognate effector (Maekawa et al. 2012). Moreover, rarl-mutant lines show reduced MLA accumulation. Yeast does have its own HSP90 homolog (SGD: S000004798), with a $63 \%$ identity to HvHSP90, though we don't know whether they are functionally redundant. Therefore, the interaction between MLA6 and SGT1 may be missing at least two necessary components. We hypothesized that by reconstituting the barley HRS complex in the Y2H system and thus restoring part of the in-planta context, we could use this to test interactions between MLA6 and SGT1.

Utilizing a Y8800 yeast strain with integrated HvRarl and HvHsp90 (Y8800:Rarl-cycl-Hsp90-adhl, Y-RcHa), we observed an increased interaction between SGT1 and MLA1 LRR fragments (Fig. 2). However, we still were unable to detect interaction with MLA6 LRR or full-length versions, suggesting that the theoretical interaction was still below an observable threshold. NLRs exhibit autoinhibition until they are activated (Ade et al. 2007; Wang et al. 2015b), therefore we hypothesized that using a fulllength autoactive mutant of MLA6 may strengthen the interaction with SGT1 to an observable level. Subsequently, by combining the Y-RcHa integrated HRS complex with the MLA6 D502V autoactive mutant, we observed an interaction between MLA6 and SGT1 (Fig. 5K2). Moreover, this interaction utilized a full-length NLR, not simply a fragment. With this data, we show that integrating cochaperones to reconstitute known complexes can be used to test interactions between proteins to be tested in $\mathrm{Y} 2 \mathrm{H}$ systems. Additionally, it may be beneficial to revisit previous experiments while using autoactive NLRs in order to identify previously unobservable interactions.

To confirm the contribution of the modified Y-RcHa strain to improving the MLA-SGT1 interaction, we utilized multiple methods for detecting the presence and expression of the integrated

Table 1. Isotopically heavy labeled synthetic peptides used in parallel reaction monitoring

\begin{tabular}{|c|c|c|c|}
\hline Peptide name & Sequence $^{a}$ & $\mathbf{M W}^{\mathbf{b}}$ & Location in sequence (aa) \\
\hline MLA6_pep1 & DPSH(L)SNLSDLVLPVK & $1,740.99$ & 784 to 799 \\
\hline MLA6.9.13_pep2 & S(L) $[\mathrm{C}(\mathrm{CAM})]$ NLHHIESLIIG[C(CAM)]NSR & $2,130.48$ & 725 to 742 \\
\hline MLA6.7.12_pep3 & WVPPVH(L)R & $1,010.22$ & 757 to 764 \\
\hline HvSGT1_pep1 & SFVESNGTV(L)STNWK & $1,675.83$ & 377 to 351 \\
\hline HvSGT1_pep2 & (L)GSYTEAVADANK & $1,345.44$ & 53 to 65 \\
\hline Control peptide ATLAS & HWYITTGPVREK & $1,486.70$ & - \\
\hline
\end{tabular}

${ }^{a}$ Parentheses surrounding a leucine $(\mathrm{L})$ indicate a heavy amino acid; [C(CAM)] indicates a carbamidomethyl cysteine.

${ }^{\mathrm{b}} \mathrm{MW}=$ molecular weight. 
genes. We did not use tagged versions of HvRarl or HvHsp90 because we were concerned that the addition of tags may interfere with their function. Therefore, we used positive PCR results from the strains to see that each gene had been stably integrated, followed by amplification and sequencing of both genes from
Y-RcHa genomic DNA. To see whether proteins were expressed, we extracted and purified peptides from diploids and performed a data-dependent acquisition MS method. We were able to detect HvHSP90 peptides from diploids derived from Y-RcHa and not from those derived from Y8800. However, we were unable to
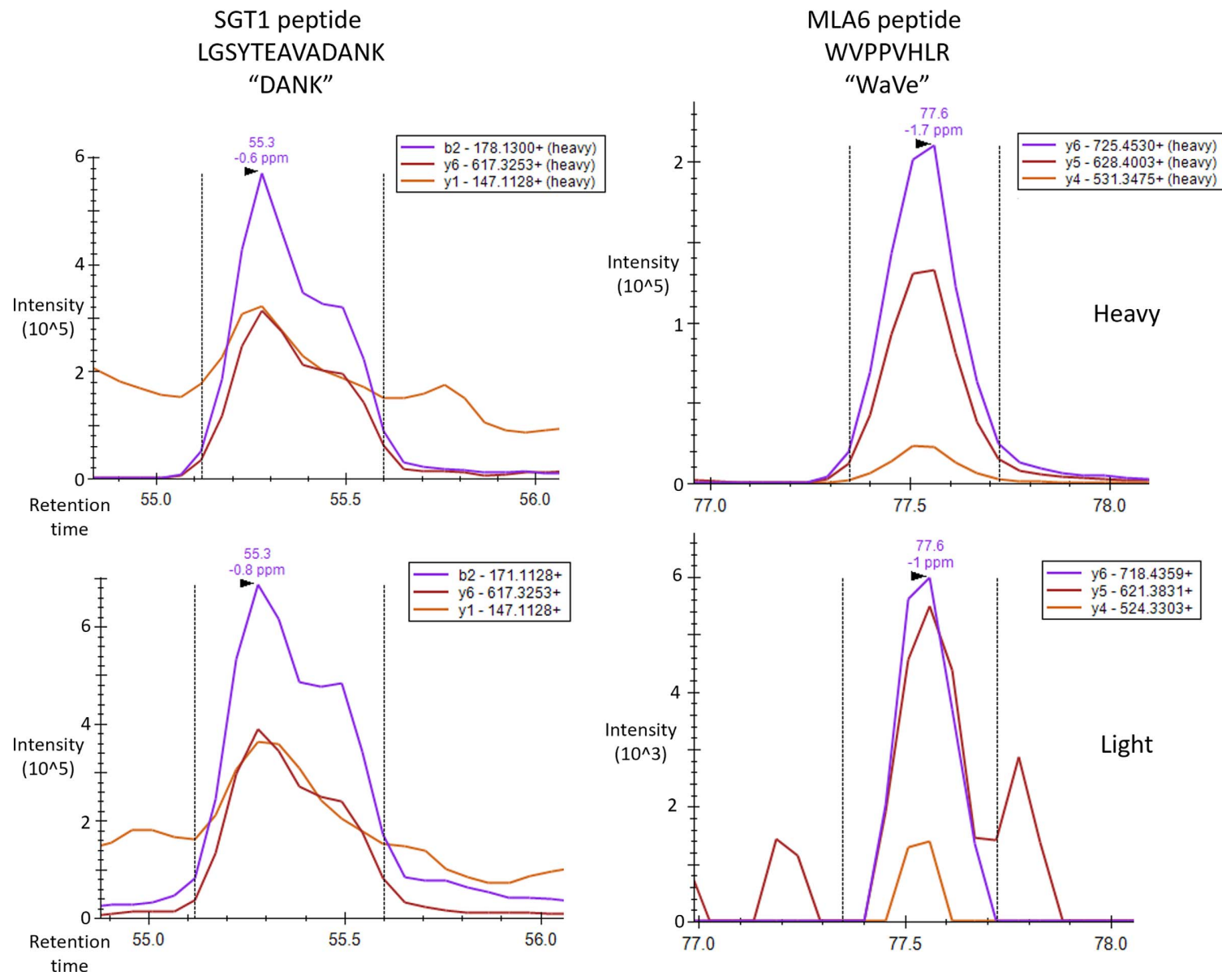

Fig. 7. Parallel reaction monitoring chromatogram results. Peak intensities for ions associated with the synthetic (top) or endogenous (bottom) versions of the SGT1 DANK peptide (left) or MLA6 WaVe peptide (right). Synthetic peptide (100 fm) was spiked into complex barley samples (1.25 $\mu \mathrm{g})$. Values above peaks indicate retention time (minutes) and mass error ( $\mathrm{ppm}$ ). Exported from Skyline.
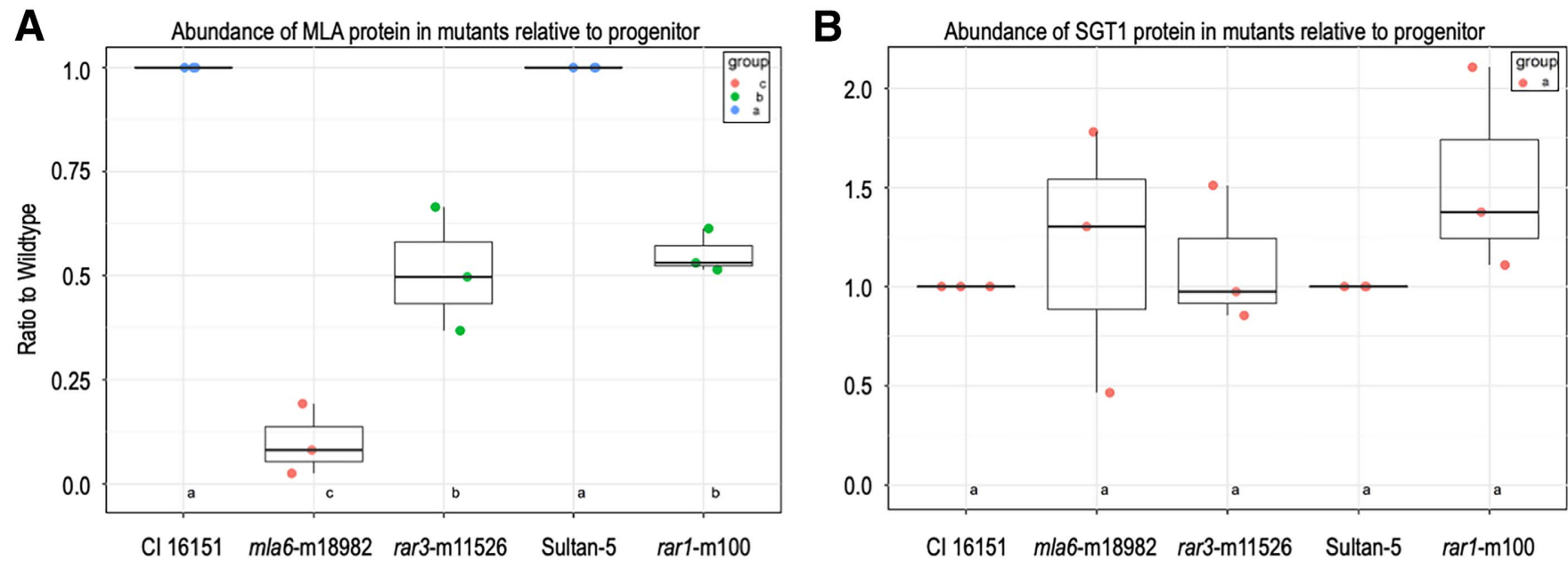

Fig. 8. Abundances of light endogenous MLA WaVe and SGT1 DANK peptides in uninfected CI 16151, Sultan-5, and derived mutants normalized to each wild-type progenitor. A, Abundance of MLA WaVe peptide. B, Abundance of SGT1 DANK peptide. CI 16151 (Mla6, Sgt1, Rar1), mla6-m18982

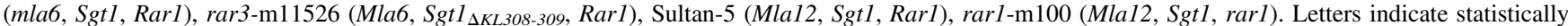
significant groups at $P<0.05$ (Supplementary Table S1). 
detect RAR1 peptides from Y-RcHa, suggesting that either the integrated Rarl may be degraded or is too low to be detected in untargeted assays.

\section{SGT1 $_{\Delta K L 308-309}$ disrupts interactions with MLA proteins.}

The recently discovered $\mathrm{rar} 3 \mathrm{Sgtl}_{\Delta K L 308-309}$ mutation alters immunity conferred by a subset of Mla alleles (Chapman et al. 2020). The $\operatorname{Sgt1}_{\triangle K L 308-309}$ mutation is in the SGS domain, which has previously been shown to interact directly with the LRR domain of MLA1, human NOD1, and the F-Box protein TIR1 (Bieri et al. 2004; da Silva Correia et al. 2007; Wang et al. 2015a). Furthermore, the deletion includes a lysine, which can be involved in protein-protein interactions through the formation of intermolecular salt bridges (Kumar and Nussinov 2002). Therefore, the $S g t 1_{\triangle K L 308-309}$ mutation may cause the loss of an interaction site between SGT1 and MLA variants.

In previous literature, an issue has been regarding which MLA variants require SGT1 function, exemplified by MLA1 and MLA6. MLA1 is considered to not require SGT1 to function, as shown by its continued function when SGT1 is silenced (Azevedo et al. 2002). However, it has also be shown to interact with SGT1 in the Y2H system (Bieri et al. 2004). In contrast, MLA6 has been shown to require SGT1, as it stops functioning when SGT1 is silenced (Azevedo et al. 2002). But prior to this study, it had not been shown to directly interact with SGT1 (Bieri et al. 2004). This begs the question, why does the variant that does not require SGT1 interact with it, whereas the variant that does require SGT1 does not? Silencing experiments have the potential to be misinterpreted, as the reduction in expression of a gene does not necessarily mean there is no protein expressed or remaining before silencing. In fact, the reason why silencing of Sgtl has been the standard for testing whether it is required for the function of other genes is because Sgtl deletions are lethal (Azevedo et al. 2006; Wang et al. 2015a). Therefore, when Sgtl is silenced, there may be some protein still performing regular function to allow the individual to survive.

The answer to the discordant requirements and interactions of MLAs and SGT1 may be that all MLA require SGT1 and interact with it, but the strength of the interaction varies and, therefore, so does their ability to retain sufficient function when SGT1 abundance is lowered. In fact, the silencing of Sgt1 does have an effect on MLA1. When Sgt1 was silenced, there was a small but not insignificant increase in susceptibility to $B$. graminis f. sp. hordei in Mlal-containing barley lines (Azevedo et al. 2006). Moreover, the loss of Rarl, which MLA1 is considered not to require, reduces MLA1 protein accumulation (Bieri et al. 2004). Thus, conditions that lower the amount of SGT1 available (via silencing) or reduce the strength of the interaction (rarl and rar3 mutations or rarl silencing) would affect the function of those MLA variants with the weaker interactions (MLA6) before they affect those with the stronger (MLA1).

We previously observed a differential effect of the $\mathrm{Sgtl}_{\triangle K L 308-309}$ mutation on MLA alleles, which may be partially explained by our results herein (Chapman et al. 2020). When we tested the interaction between MLA1 543-968 and SGT1 WT using Y8800, we found that it was relatively weak and it was difficult to see a significant difference between the interac-

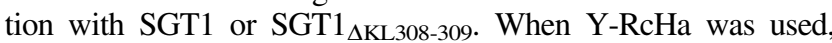
the interaction between MLA1 and SGT1 WT was stronger but we saw no obvious change in the strength of the interaction between MLA1 and SGT1 ${ }_{\Delta \mathrm{KL} 308-309}$ in the Y-RcHa environment. This suggests that the SGT1 $1_{\Delta \mathrm{KL} 308-309}$ mutation does interrupt the interaction between MLA1 and SGT1 but only to a comparable level as lacking one or both RAR1 and HSP90. Moreover, when we used the autoactive mutant MLA6 $6_{\mathrm{D} 502 \mathrm{~V}}$, which did interact with WT SGT1, we still did not observe an interaction with $\mathrm{SGT}_{\Delta \mathrm{KL} 308-309}$. Furthermore, we were able to disrupt this interaction further by using the $\mathrm{SGT} 1_{\mathrm{K} 308 \mathrm{R}}$ and SGT1 $1_{\mathrm{KL} 308 \mathrm{AA}}$ mutants, leading to complete loss of observable interaction with MLA1. Together, these results suggest that the $\mathrm{Sgt1}_{\triangle K L 308-309}$ mutation does not lead to complete loss of SGS function but, rather, an abating of its function.

$\mathrm{SGT}_{\Delta \mathrm{KL} 308-309}$ could be tested for interactions with other proteins that interact with the SGS domain to test whether this weaker interaction is uniform or specific. There are a few possible outcomes of these tests; SGT1 $1_{\Delta \mathrm{KL} 308-309}$ may cause an altered interaction with only MLA, suggesting that this is a site specific to MLA interactions or $\mathrm{SGT1}_{\Delta \mathrm{KL} 308-309}$ may cause a weakened interaction with all NLR interactors but not with other types of interactors, which would suggest that this site is specific to disease resistance pathways. Another possibility is that $\mathrm{SGT}_{\Delta \mathrm{KL} \text { 308-309 }}$ may disrupt interactions with all proteins that
A

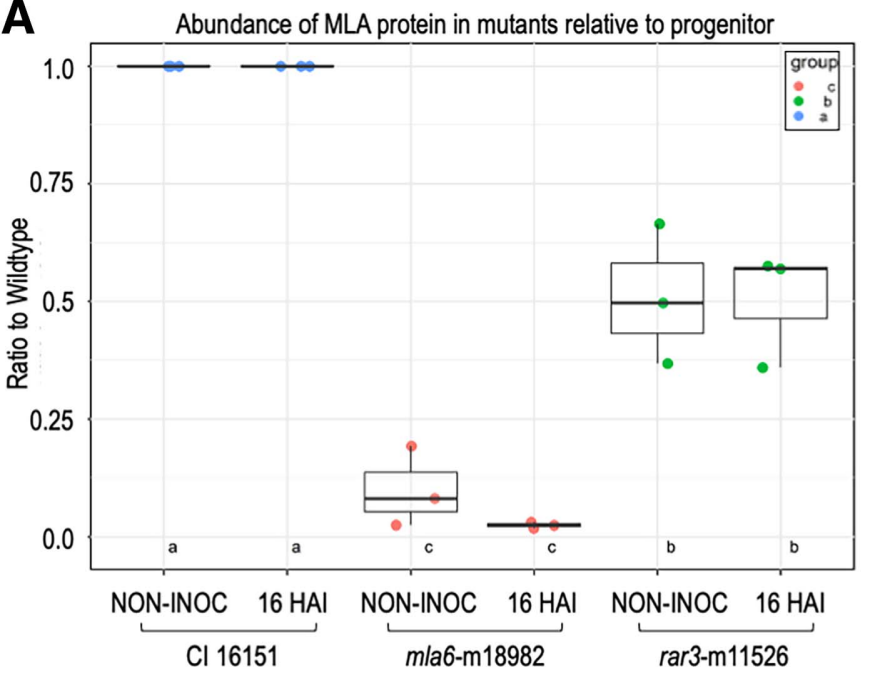

B

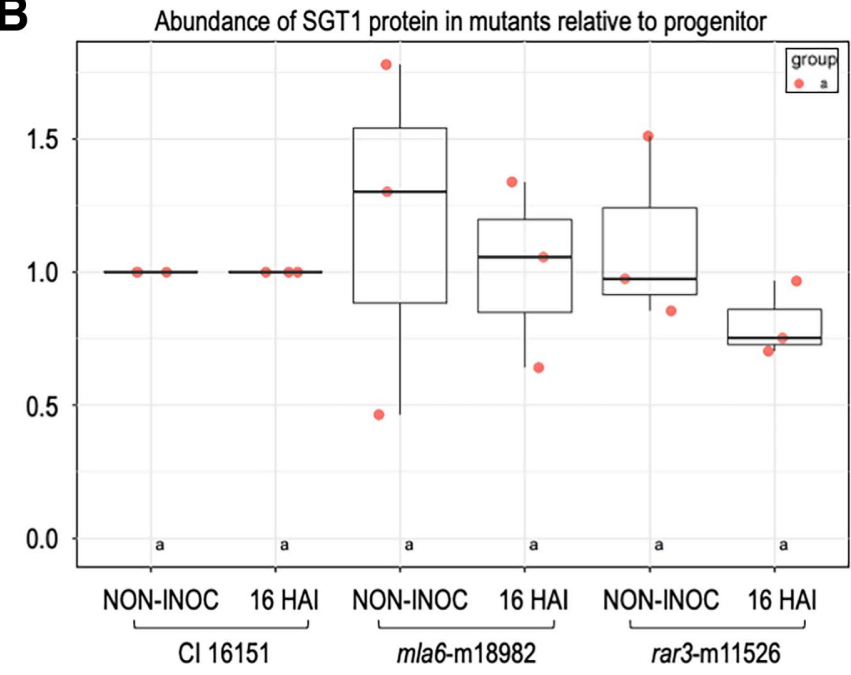

Fig. 9. Abundances of light endogenous MLA WaVe and SGT1 DANK peptides of CI 16151 and derived mutants in uninfected plants and plants 16 $\mathrm{h}$ after inoculation (HAI) normalized to the wild-type progenitor. A, Abundance of MLA WaVe peptide. B, Abundance of SGT1 DANK peptide. CI 16151 (Mla6, Sgt1, Rar1), mla6-m18982 (mla6, Sgt1, Rar1), rar3-m11526 (Mla6, Sgt1 ${ }_{\triangle K L 308-309,}$ Rar1). "NON-INOC" indicates noninoculated, uninfected samples, "16 HAI" indicates samples $16 \mathrm{~h}$ after inoculation with $B$. graminis f. sp. hordei isolate $5874\left(A V R_{a 6}\right)$. Letters indicate statistically significant groups at $P<0.05$ (Supplementary Table S1). 
interact with the SGS domain, but it is a small reduction that we only see manifest in a subset. This last possibility is given credence by the fact that resistance pathways appear to exist in a heavily regulated equilibrium just below the threshold required for activation, so that they are able to signal quickly upon detection of a pathogen but do not cause rampant autoimmune responses (Bentele et al. 2004; Bernoux et al. 2016). Therefore, if SGT1 ${ }_{\Delta \text { KL308-309 }}$ only slightly reduces interactions, it may significantly shift the equilibrium, causing a loss of action by the NLRs with the less robust interactions.

\section{Y2H assays indicate $S G T 1_{\text {KL308-309 }}$ is important for SGT1-MLA interactions.}

We used SGT1 mutant constructs to investigate why the $\mathrm{Sgt1}_{\triangle K L 308-309}$ mutation affects its function in disease. We found that $\operatorname{Sgt1}_{\triangle K L 308-309}$ does appear to slightly alter its ability to interact with MLA1. However, the destabilization caused by $\mathrm{Sgtl}_{\triangle K L 308-309}$ is not as severe as the double alanine substitution or K308R (Fig. 3). An interesting conundrum is that the SGS domain of SGT1 interacts with a multitude of unrelated NLRs. Moreover, it interacts with the LRR domain, which is the most diverse part of the NLRs. For the SGS domain to interact with a multitude of varied LRRs, either it must have extreme flexibility, there are shared LRR motifs that it interacts with, or linker proteins bridge the gap, as suggested by Kadota et al. (2010). Considering the extreme disorder of the SGS domain, we hypothesize that extreme flexibility is the most likely. It is possible that the SGS domain has the ability to interact with a multitude of proteins because it adopts different conformations, allowing its variable binding sites to interact with LRRs where possible. This is supported by our findings that the diminished interaction caused by the $S g t 1_{\Delta K L 308-309}$ deletion, which arguably would increase disorder, was less severe than alanine mutations or the K308R mutation, which are predicted to decrease disorder.

We examined the structure of barley SGT1 to look for clues as to why the $S g t 1_{\triangle K L 308-309}$ deletion may affect its function (Taube et al. 2014). The SGS domain consists of three coils and unstructured regions (Fig. 10). In the SGS domain, almost $40 \%$ of the amino acids are either aspartate (D, about $11 \%)$, glutamate $(\mathrm{E}$, about $11 \%)$, or lysine (K, about $17 \%)$, all of which can be involved in forming intermolecular salt bridges. In coil 1, where the $\operatorname{Sgt}_{\triangle K L 308-309}$ mutation is located, the proportions are even higher, D about $18 \%, \mathrm{E}$ about $18 \%$, and $\mathrm{K}$ about $32 \%$, totaling almost $70 \%$ of the residues. Moreover, when these three residues are mapped onto the three-dimensional structure, all the lysines appear on the same coil side (Fig. 10B). This may indicate this side has potential to form multiple salt bridges with interacting proteins, perhaps with a domain with many surface aspartates and glutamates. Interestingly, there is a distinct lack of arginine, both in the whole SGS domain (about 3\%) and coil $1(0 \%)$, despite its function in forming salt bridges, which suggests that there may be some alternate characteristic that make its unsuitable for this domain. This negative selection may indicate why the K308R mutation has such a drastic affect.

\section{A reduction in the strength of interaction with SGT1 correlates with a lower MLA abundance.}

The protein RAR1 is a cochaperone of SGT1 that is required for the function of some MLA proteins, such as MLA6 and MLA12, but not for others, such as MLA1 and MLA7 (Halterman and Wise 2004). Though it is considered to not be required for MLA1 function, loss of RAR1 has been shown to reduce the abundance of MLA1 and MLA6 (Bieri et al. 2004). As m11526 has a similar phenotype to rarl mutants, we hypothesized that MLA abundances may be lowered as a result of the $S g t 1_{\triangle K L 308-}$ 309 mutation. To pursue this, we utilized PRM MS to assay MLA in native CI 16151 and derived mutant plants. This method was selected as it displays higher sensitivity to low abundance peptides (Gallien et al. 2012) and MLA6 peptides have not been detected using other MS methods (Lambertucci et al. 2019).

Using an adapted PRM method, we observed previously unrecorded endogenous peptides for MLA6, MLA12, and SGT1 (Fig. 7). We observed that in 11526 (Mla6, Sgt1 ${ }_{\Delta K L 308-309}$, Rarl), the steady-state levels of MLA6 were much lower than in the WT progenitor CI 16151(Mla6, Sgt1, Rarl) (Fig. 8). This is especially significant considering that accumulation of Mla6 transcripts is not significantly different between $\mathrm{m} 11526$ and CI 16151 before or during infection (Chapman et al. 2020). In contrast, there was no significant difference in the abundance of SGT1 in any line tested (Fig. 9), which corresponds to previous studies (Azevedo et al. 2006; Noël et al. 2007). This reduction of MLA6 abundance in m11526 does not equate to the complete loss of abundance in the phenotypically similar mla6 deletion mutant m18982 (mla6, Sgtl, Rarl). This lends credence to the hypothesis that resistance proteins have a threshold level that is necessary for them to function. The relative abundance of MLA6 in the $S_{g t 1_{\Delta K L 308-309}}$ mutant line was consistently lower even after challenge with $B$. graminis f. sp. hordei 5874 (Fig. 10), which suggests a consistent effect of the mutation upon protein levels.

Interestingly, in the line Sultan-5 (Mla12, Sgt1, Rar1), MLA12 accumulated roughly twice as much as MLA6 in CI 16151 (Supplementary Fig. S4A). In contrast, in the rarl susceptible mutant m100 (Mla12, Sgt1, rarl), MLA12 abundance levels were much lower than the WT progenitor Sultan-5, to levels equal to the $\mathrm{Sgt1}_{\triangle K L 308-309}$ mutant line. This may suggest that the thresholds for functionality differ greatly between different MLA variants, though an abundance reduction of about $50 \%$ is enough to lose functionality. Or it may indicate that Sultan-5 has other factors that reduce the effectiveness of harbored MLA alleles. Therefore, a higher abundance of MLA12 may be required to perform the same function as MLA6.

This data corresponds with previous findings that MLA1 accumulated to much higher levels than MLA6, even when these levels were greatly reduced by the rarl mutation (Bieri et al. 2004). However, the MLA1 phenotype was unaffected by the large reduction of abundance, unlike the susceptibility caused in $\mathrm{m} 100$ by the rarl mutation. Together, these data suggest that the $S g t 1_{\Delta K L 308-309}$ mutation causes susceptibility in m11526 by reducing the abundance of MLA6 to a level below the threshold required for effective resistance.

\section{A new cyclical model for MLA-HRS interactions.}

To generate a new hypothetical model of MLA-HRS interactions, we combined our data with recent discoveries in NLRs. The NB domain of NLRs bind either ATP or ADP to act as an intramolecular switch, where the ADP bound state is inactive, and the ATP bound state is active (Williams et al. 2011). Moreover, NLRs appear to naturally flit between these ATP and ADP bound forms outside of pathogen recognition, in part due to inbuilt ATP hydrolysis (Bernoux et al. 2016; Marquenet and Richet 2007). However, it has been shown that the active ATPbound state is kept at a very low level, possibly to reduce autoactivation (Bernoux et al. 2016). Interestingly, the flax L protein in the active ATP-bound state binds its matching effector more stronly than the inactive version (Bernoux et al. 2016), and similarly, here, we show that autoactive MLA proteins interact with SGT1 more strongly than respective WT variants. Moreover, there is recent evidence that the interaction between NLRs and ligands stabilizes the active conformations of NLRs (Yang et al. 2018). As dimerization of CC domains are required for signaling (Maekawa et al. 2011) and the HRS complex is predicted to allow docking of two NLRs simultaneously (Siligardi et al. 2018), then the HRS may facilitate this dimerization. Therefore, the model proposed by (Bernoux et al. 2016), which 
describes an NLR being locked in its active state by an effector, can be expanded to include the interaction with the HRS complex.

We postulate that SGT1 may exist in a constant state of briefly interacting with ephemerally activated NLRs (Fig. 11A). MLA6, in its free, inactive ADP-bound state will spontaneously swap ADP for ATP, this releases autoinhibition and "activates" the NLR. Once active, MLA6 can dock with the HRS. However, MLA6 may automatically hydrolyze the ATP into ADP, which will cause MLA6 to form back into its autoinhibited state and eject from the HRS. This completes the active/inactive cycle. The small pool of ATP-bound NLR observed by Bernoux et al. (2016) suggests that the ATP-bound state is very brief, which could indicate that the spontaneous activation of an NLR may occur relatively infrequently compared with the rest of the cycle. However, if all the NLR proteins are cycling between states, then it is likely that there is always a small pool of active and docked NLRs maintained.

If a pathogen introduces an effector into the cell during infection (Fig. 11B), then, during the brief window of MLA6 being active and docked with the HRS, the cognate effector can interact with the MLA6 LRR domain. This interaction stabilizes it in the active state by inhibiting ATP hydrolysis, preventing disassociation from the HRS. This provides the opportunity for another MLA6 unit to dock with the HRS and become stabilized. Once two MLA6s are docked and stabilized, the CC domains are held in proximity, promoting homomeric dimerization and initiating the defense-signaling cascade. This model is compatible with any NLR that requires the HRS complex and functions in a homo- or heteromeric pair. Therefore, for NLRs such as MLA6, the MLA6-HRS-effector complex could be considered the "resistosome".

The MLA6-HRS-effector model is an alternative mode of action to the recently described resistosome complexes for other NLR proteins. Both CC-NLRs and toll interleukin 1-NLRs have been found to dimerize by way of their $\mathrm{N}$-terminal domains like MLA (Casey et al. 2016; Huber et al. 2015; Maekawa et al. 2011; Zhang et al. 2017). However, others such as ZAR1 and RPP7, have been found to form larger multimeric pore-like structures, termed resistosomes, which do not require SGT1 or RAR1 to function (Lewis et al. 2010; Li et al. 2020; Wang et al. 2019a). So far, this appears to represent a key difference between many NLRs, either they oligomerize into groups of four or more and do not require the HRS or they function in dimeric pairs and do require the HRS. Therefore, as the HRS complex is predicted to be able to dock two NLRs simultaneously (Siligardi et al. 2018), it is possible that the HRS confers the dimeric constraint on NLRs that it requires to function. This model can fit with the observation of lower abundance of MLA protein in either the $H v S g t 1_{\triangle K L 308-309}$ or rarl mutant lines. In line with this hypothetical model, when MLA6-HRS docking is compromised for whatever reason, such as competition or a mutation ( $r a r l$ or rar3), then active-state MLA6 would be prone to degradation instead of recycling back into the inactive state. Thus, the abundance of MLA protein in these mutants would be lower without additional environmental factors, such as pathogen exposure.

\section{Conclusion.}

Our investigation of the $H v S g t 1_{\Delta K L 308-309}$ mutation adds a new layer onto that of previous studies by presenting multiple new ways to study the relationship between SGT1 and NLR proteins. Due to being unable to make $S g t l$ knock-out mutants, the requirement of $S g t 1$ for $R$ genes to function has historically been performed by silencing experiments (Fu et al. 2009; Kud et al. 2013; Liu et al. 2016; Peart et al. 2002). However, not only are Sgtl-silencing experiments in $N$. benthamiana called into question by recent studies (Yu et al. 2019), but our results suggest that NLRs that are able to function despite the silencing of Sgt1 may, rather, be those that interact most strongly with $\mathrm{Sgt}$.

The HRS complex is the core for many eukaryotic cell processes. However, inter- and intramolecular interactions for this complex are only partially understood. For instance, Kadota and Shirasu (2012) stated that, for us to understand how RAR1 causes SGT1 to interact more strongly with NLRs, we need to understand how the SGT1 SGS domain interacts with the LRR domain of NLRs. Due to the lack of an interaction between MLA6 and SGT1, which MLA6 requires to function, it had been proposed that linker proteins may be involved, such as HSP70 (Kadota et al. 2010). However, by reconstituting the HRS complex in our Y2H system and by using an autoactive full-length MLA6, we provide evidence that MLA6 and SGT1 can interact when we mimic the in-vivo context in which these proteins would need to interact. Mutant MLA constructs, generated by examining correlations in amino acid sequence between groups either disrupted by the $S g t 1_{\triangle K L 308-309}$ mutation or not, suggest that there are key positions in the LRR domain that are required for this interaction to occur. Moreover, the effect of mutant SGT1 constructs on interactions with MLA suggest that the exceptionally disordered nature of the SGS domain may be important for its function by allowing it to adapt to the variety of protein interactors.

Though the HvSgt1 ${ }_{\triangle K L 308 \text {-309 }}$ mutation disrupts the interaction between MLA and SGT1, the lethality or stunting that usually
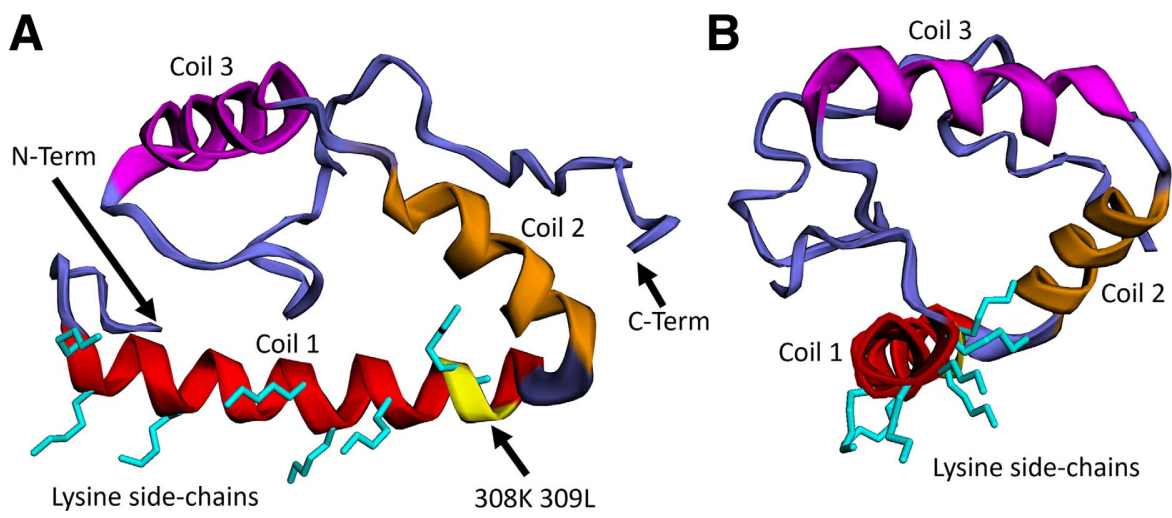

Fig. 10. Structure of HvSGT1 SGT1-specific (SGS) domain. Generated from data generously provided by (Taube et al. 2014) and modeled in EZMol v2.1. A, The SGS domain spans from amino acid (aa) 285 to 373. Coil 1 (red) spans aa 290 to 311, coil 2 (orange) spans aa 314 to 325 , coil 3 (fuchsia) spans aa 328 to 341, unstructured regions are shown in blue. The N-terminal connects to the VR2 domain, and the C-terminal is the terminus of the protein. Lysine side chains in coil 1 are represented by stick models (teal). Positions K308 and L309 in coil 1 are shown in yellow. B, Side view of the HvSGT1 SGS coil 1 showing that all the lysine side chains in coil 1 appear on one half of the coil. 
occurs when Sgtl is mutated or silenced is absent. This indicates that the abating still allows most cell processes to proceed as normal, while only disrupting the weakest interactors of SGT1. This permits us a unique opportunity to study Sgtl in disease resistance isolated from confounding factors. Reciprocal mutations made in other crops may answer similar questions regarding the requirement of Sgtl for NLRs to function. It is possible that the essential protein, SGT1, is required by most CC-NLRs in some capacity and, thus, is important for further study.

\section{MATERIALS AND METHODS}

\section{Biological materials.}

Seven-day-old barley seedlings of CI 16151 (Mla6, Manchuria background), its fast-neutron derived mla6 (m18982) and rar3 (m11526) deletion mutants (Chapman et al. 2020), and P04B (Mla7, Pallas background) (Saur et al. 2019) were infected with B. graminis f. sp. hordei isolate 5874. At $24 \mathrm{HAI}$, first leaves of these seedlings were harvested, were flash-frozen in liquid nitrogen, and were stored at $-80^{\circ} \mathrm{C}$ before RNA was

A

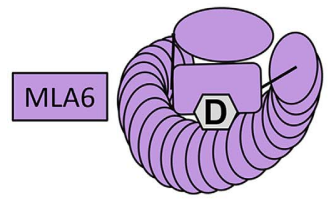

$\underline{\text { Inactive/active cycle }}$

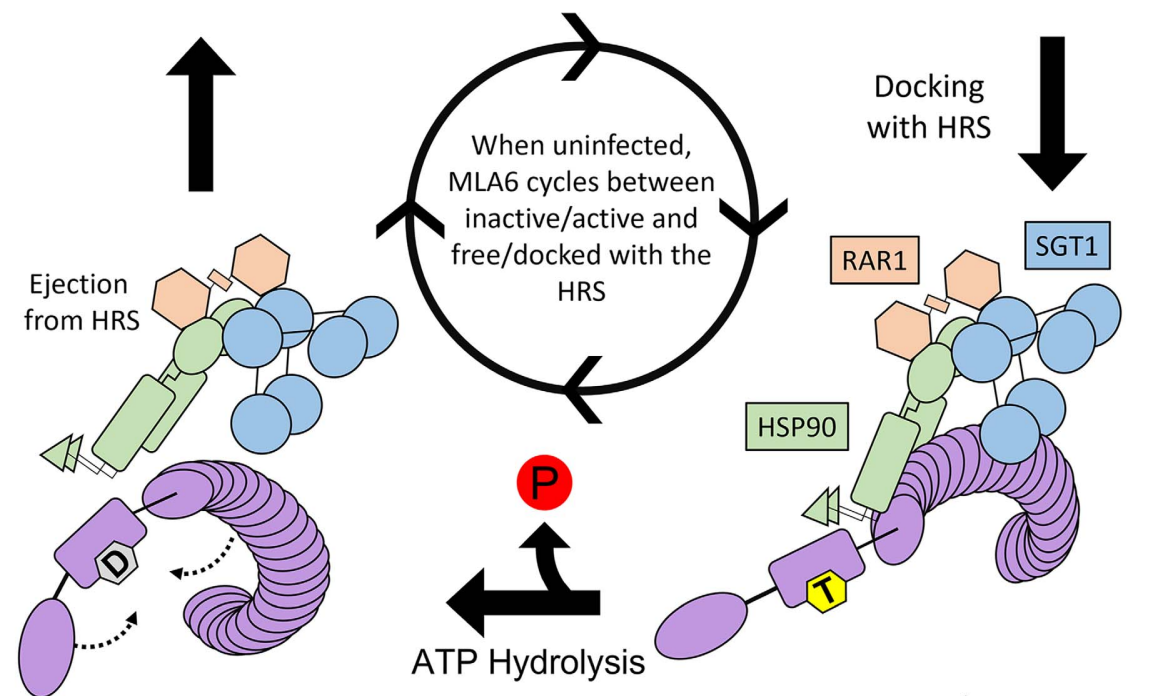

B

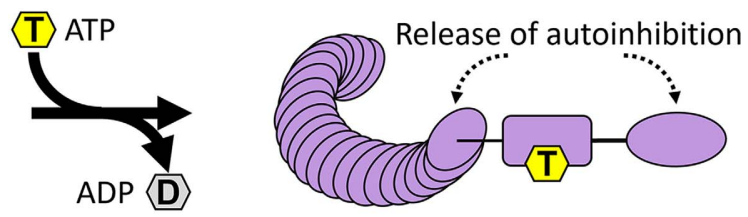

ATPHydrolysis

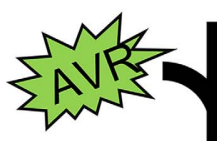

When infected, pathogen introduces cognate effector (AVR) that interacts with active/docked MLA6 to inhibit ATP hydrolysis

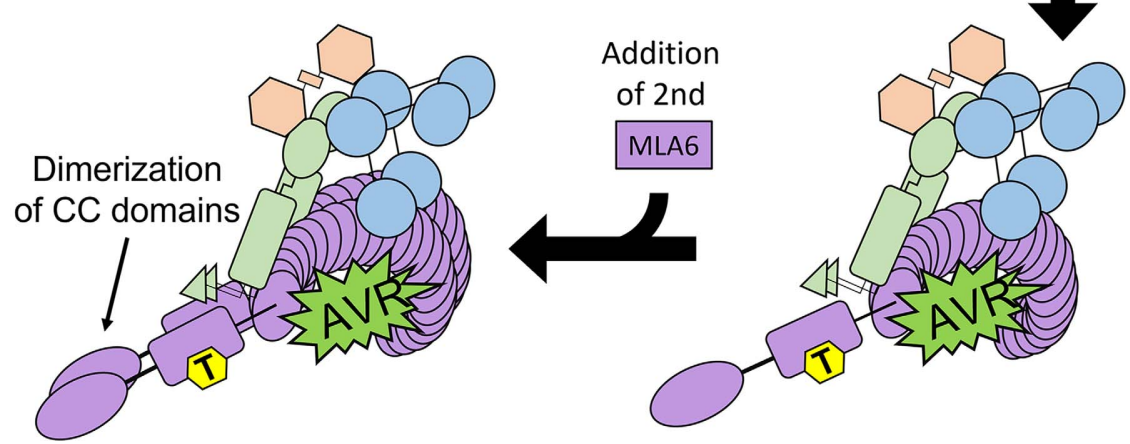

Fig. 11. MLA6-HRS (HSP90, RAR1, and SGT1) activation cycle model showing interactions between MLA6, the HRS, and pathogen effectors. A, The inactive/active cycle. MLA6 (pur ple) primarily exists in an ADP-bound autoinhibited state. ADP is spontaneously swapped for ATP, which stops MLA6 autoinhibiting. Released from autoinhibition, MLA6 can dock and interact with the HRS, competing with other cofactors. Without the involvement of other factors, MLA6 will eventually hydrolyze the ATP into ADP, which causes it to eject from the HRS complex. Once freed, it reverts to its autoinhibited state. The smaller arrow for the swapping of ADP for ATP represents that this process likely happens at a slower rate than the rest of the model. B, Effector stabilized switch. When a pathogen introduces the cognate effector of the MLA6 (AVR, green), it will interact with the leucine-rich repeat domain of MLA6 and inhibit ATP hydrolysis, stabilizing the complex in the active conformation. This provides opportunity for another MLA6 to dock with the same HRS and also be stabilized by the effector. With two MLA6 docked and stabilized with the HRS, the CC domains will be able to dimerize and initiate defense response signaling. 
isolated as described (Caldo et al. 2004). cDNA synthesis was performed with SuperScript III reverse transcription (Invitrogen, ThermoFisher). The P04B allele of Mla7 was chosen over the allele present in the line CI 16147 (Manchuria background, Mla7) due to its stronger cell-death phenotype (Saur et al. 2019).

$N$. benthamiana seeds were germinated on the surface of soil for 7 to 10 days before being transplanted to 3 -inch pots. Agrobacterium tumefaciens EHA105 was used for the transient expression of all constructs used in $N$. benthamiana, including pEG100 and pHELLSGATE8 (pHG8).

\section{Experimental constructs.}

pEarleyGate100 (pEG100, Kan 50, ABRC stock CD3-724) was used to overexpress proteins of interest in $N$. benthamiana, under control of the cauliflower mosaic virus $35 \mathrm{~S}$ promoter. Genes were first amplified from barley cDNA and inserted into the gateway entry pCR8 TOPO TA backbone (ThermoFisher) and were then inserted into the appropriate vector via Gateway LR reactions, $\mathrm{pEG} 100$, $\mathrm{p} 97-\mathrm{BD}$, or $\mathrm{p} 86-\mathrm{AD}$.

Silencing constructs targeting $N$. benthamiana Sgtl (NbSgt1) were designed by aligning the available $\mathrm{NbSgtl}$ sequences (AY899199, AF494083, AF516180, and AF516181) and amplifying the most conserved regions at $<600$ bp with PCR from cDNA (primers are provided in Supplementary Table S2). Though the amino acid sequence is conserved between $N$. benthamiana and barley, the nucleotide sequences are unconserved with no consecutive matching bases longer than an outlier of $19 \mathrm{bp}$, which is below the 20-bp limit for hairpin formation. The amplicon of 78 to $586 \mathrm{bp}, 509 \mathrm{bp}$ long, was then inserted into the hairpin silencing construct pHG8 (Helliwell and Waterhouse 2003).

\section{MS sample preparation.}

For each genotype, CI 16151 (Mla6, Sgt1), mla6-m18982

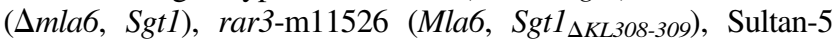
(Mla12, Rar1), and rarl-m100 (Mla12, rar1-2), three biological replicates of six seeds were planted in 3-inch pots, randomly placed in 16-cell flats. Of the six seed planted, five uniform leaves were collected at 7 days or at 7 days plus 16 HAI with $B$. graminis f. sp. hordei and were frozen immediately in liquid nitrogen for storage prior to processing. Leaves were then ground to a fine powder, in liquid nitrogen, using a mortar and pestle for $15 \mathrm{~min}$ per sample, maintaining a small pool of liquid nitrogen to preserve sample quality during grinding.

Protein was extracted from barley tissue using a phenol extraction method, was processed with filter aided sample preparation, which included digestion with trypsin and Lys-C, and was cleaned up, using $\mathrm{C} 18$ cartridges, as described by Song et al. (2018). After processing, samples were resuspended in $0.1 \%$ formic acid (FA) and were quantified using a Pierce BCA protein assay kit (ThermoFisher).

\section{Media.}

Escherichia coli were grown on Luria-Bertani agar with the appropriate antibiotics. Yeast was grown on yeast peptone dextrose or SC- (synthetic complete dropout) media (Burke et al. 2000).

\section{Y2H system.}

The Y2H system (Dreze et al. 2010) utilizes yeast strains Y8800 MATa and Y8930 MAT $\alpha$ (trp1-901; leu2-3, 112; ura352; his3-200; gal4D; gal80D; GAL2-ADE2; LYS2::GAL1-HIS3; MET2::GAL7-lacZ; cyh $2^{R}$ ). Transformation of yeast with plasmids was conducted using the LiAc/SS carrier DNA/PEG method (Gietz 2014). The plasmids pDEST-DB (also known as pPC97 or p97-BD, Leu2p) and pDEST-AD (also known as pPC86 or p86-AD, Trplp) are low-copy ARS4/CEN6-based that encode for fusions to the GAL4 DNA-binding domain (DB) and GAL4 activating domain (AD), respectively. pDEST-DB and pDEST-AD allow for the growth of Y8800 or Y8930 on media lacking leucine (SC-L) or tryptophan (SC-W), respectively. Selection for Y8800-Y8930 diploids harboring both pDEST-AD and pDEST-DB was done by growing on SCmedia lacking both leucine and tryptophan (SC-LW). When the GAL4 AD and DB are in close proximity, caused by an interaction between their fused proteins, they induce expression of HIS3 by associating with the 125-bp GAL1 upstream activating sequence that has been inserted in the promoter region. The degree of interaction between the proteins fused to the GAL4 $\mathrm{AD}$ and $\mathrm{DB}$ domains was estimated by the level of growth of the diploid on media lacking histidine (SC-LWH), a higher level of growth indicating a more effective interaction.

\section{Stable yeast introgression.}

The yeast strain Y8800 was engineered to stably express HvRAR1 and HvHSP90 using the system developed by Mikkelsen et al. (2012). The yeast promoter TEF1 from Y8800 was isolated, using PCR, from extracted genomic DNA (Lõoke et al. 2011) with an added AsiSI restriction enzyme site on the 3 ' end of the reverse primer (Supplementary Table S2 provides a list of primers). The TEF1 promoter was inserted into plasmids pXI-5 and pXII-3, oriented to the ADH1 and CYC1 terminators, respectively, creating the constructs pXI-5-TEF1-ADH1 and pXII-3-TEF1CYCl. HvRarl (HORVU2Hr1G097800) Morex v1 and HvHsp90 (HORVU5Hr1G072420) Morex v1 were amplified from barley cDNA and were inserted separately into both constructs.

Yeast strain Y8800 was transformed with each of the four constructs and was then selected on SC-uracil plates for successful transformation events, indicated by presence of the URA3 marker, and presence of the barley gene was confirmed by PCR amplification. The URA3 marker is designed to excise out of the yeast genome by direct repeat recombination to allow for multiple uses in the same strain. Colonies of transformed Y8800 with the URA3 gene excised were selected for by growth on SC media containing uracil and 5-fluoroorotic acid (ThermoFisher), which is lethal to strains with a functional URA3 gene. Loss of URA3 marker gene was confirmed by a lack of growth on SC-uracil media. Strains that were successfully engineered with a single gene and that lost the URA3 marker were transformed again with the complementary second gene and were selected by the same method as described for the first transformation. For example, Y8800-XII-3Rar1-CYC1 was transformed with pXI-5-TEF1-HSP90-ADH1. This resulted in one strain (Y8800-XII-3-Rarl-CYC1-XI-5$H$ sp90-ADH1, also known as Y-RcHa, nicknamed Y archer) that had both HvRarl and HvHSP9O integrated into the genome on chromosomes XII and XI, respectively, and with different terminators ( $\mathrm{CYC1}$ and $\mathrm{ADH} 1$, respectively). This strain was transformed with pDEST-AD plasmids to generate Y-RcHa strains for use in $\mathrm{Y} 2 \mathrm{H}$. A diagram of the process is outlined in Supplementary Figure S5.

\section{Mutagenesis.}

We performed site-directed mutagenesis using the Quikchange Lightning site-directed mutagenesis kit (Agilent, Santa Clara, CA, U.S.A.) per the manufacturer guidelines. Primers used for mutagenesis are listed in Supplementary Table S2.

\section{Peptide design.}

Peptide design was conducted following guidelines set down by Rauniyar (2015). Peptides for MLA6 and SGT1 were designed using SkyLine software under a trypsin digest (Pino et al. 2020). Candidate peptides were searched using BLASTp against the barley protein database on EnsemblPlants (IBSC v2) to look for uniqueness. PEPOtec grade 2 synthetic versions of these peptides with heavy leucines were ordered from ThermoFisher) (quality 
Table 2. Inclusion list used for parallel reaction monitoring of synthetic heavy and endogenous light versions of SGT1 DANK and MLA6 WaVe ${ }^{\text {a }}$

\begin{tabular}{|c|c|c|c|c|c|c|c|c|}
\hline $\operatorname{Mass}(m / z)$ & Formula (M) & Species & CS $(z)$ & Polarity & Start (min) & End (min) & NCE & Comment \\
\hline 669.830431 & & & 2 & Positive & & & 28 & LGSYTEAVADANK (light) \\
\hline 673.339013 & & & 2 & Positive & & & 28 & LGSYTEAVADANK (heavy) \\
\hline 502.295436 & & & 2 & Positive & & & 28 & WVPPVHLR (light) \\
\hline 505.804018 & & & 2 & Positive & & & 28 & WVPPVHLR (heavy) \\
\hline
\end{tabular}

${ }^{\mathrm{a}}$ For direct import to use in Excalibur, empty spaces are left blank intentionally, as this format is required by the software. CS $=$ charge state and NCE $=$ normalized collision energy.

certificates are provided in Supplementary Data S1). Upon receipt, synthetic peptides were resuspended in $0.1 \% \mathrm{FA}$ and working stocks were made at a concentration of $1,000 \mathrm{fmol} / \mu \mathrm{l}$.

\section{PRM liquid chromatography (LC)-MS method.}

LC-MS was performed using an Ultimate 3000 RSLCnano HPLC coupled to a Q-Exactive Plus mass spectrometer with a nano-electrospray ion source (ThermoFisher). Capillary columns $(100 \mu \mathrm{M}$ ID $\times 25 \mathrm{~cm})$ were pulled to an approximately $10 \mu \mathrm{M}$ tip with a laser puller (Sutter P-2000) and packed with approximately $1 \mathrm{~cm}$ of $5 \mu \mathrm{M} \mathrm{C} 18$ particles (Agilent) at the tip and were then filled up to the full length with $2.5 \mu \mathrm{M} \mathrm{C} 18$ particles (Waters, Milford, MA, U.S.A.). Peptide samples (10 $\mu \mathrm{l}[1,250 \mathrm{ng}])$ in buffer A $(0.1 \%$ formic acid $(\mathrm{vol} / \mathrm{vol}))$ were injected, using an autosampler with a $20-\mu \mathrm{l}$ sample loop, and were preconcentrated on a $0.3 \times$ $5 \mathrm{~mm}$ trap cartridge (ThermoFisher) for $5 \mathrm{~min}$ at $10 \mu \mathrm{l} / \mathrm{min}$, using the loading pump. At $5 \mathrm{~min}$, the trap column was switched in-line with the nano-capillary pump set at $1 \%$ buffer B $(0.1 \%$ FA [vol $/ \mathrm{vol}$ ], $80 \%$ acetonitrile [ $\mathrm{vol} / \mathrm{vol}]$ ) at a flow rate of $250 \mathrm{nl} / \mathrm{min}$. The 150-min LC program consisted of a gradient from 1 to $6.3 \%$ buffer B at 4 to $5 \mathrm{~min}$, followed by 6.3 to $31.3 \%$ for 5 to $110 \mathrm{~min}$, then 31.3 to $56.3 \%$ for 110 to $135 \mathrm{~min}$, then $99 \%$ at 135.5 to $140.5 \mathrm{~min}$, and reduced to $1 \%$ buffer B at $141 \mathrm{~min}$.

For PRM, the MS program consisted of a full MS scan followed by four DIA (data independent acquisition) scans using an inclusion list (Table 2). The full MS scan was acquired at a resolution of 70,000, AGC target 1e6, maximum injection time (IT) of $50 \mathrm{~ms}$, and scan range of 150 to $2,000 \mathrm{~m} / \mathrm{z}$. The DIA scans used a resolution of 140,000, AGC target of $3 \mathrm{e} 6$, maximum IT of $500 \mathrm{~ms}$, loop count of 4, isolation window of $0.4 \mathrm{~m} / \mathrm{z}$, and normalized collision energy of 28 . The optimal sample for detection of the peptides was $1.25 \mu \mathrm{g}$ of complex samples were run with synthetic peptides added at an amount of $200 \mathrm{fm}$ for SGT1 DANK and $10 \mathrm{fm}$ for MLA6 WaVe.

\section{Data analysis.}

Raw output files from Xcalibur for each run were imported into Skyline (Pino et al. 2020) (version 20.1.0.155). Peptide settings were as shown in Supplementary Figure S6. The background proteome was the barley proteome downloaded from EnsemblPlants, ISBC v2. The reference library was generated from the .raw file PXD012684 downloaded from Proteome Exchange (Lambertucci et al. 2019).

\section{Peptide detection.}

The synthesized peptides were first run on their own, in order to evaluate the quality of the peptides and the suitability of the method at amounts ranging from 1 to $1,000 \mathrm{fm}$. The resulting chromatograms were compared with results from a publicly available barley MS dataset (Proteome Exchange PXD012684) (Lambertucci et al. 2019), though differences in equipment and settings cause expected variability between studies. Besides the ATLAS control peptide, only two of the five synthesized peptides were consistently observed, MLA6.7.12_Pep3 WaVe and HvSGT1_Pep2 DANK (Fig. 7). These two peptides were then spiked into complex WT uninfected barley samples to look for the endogenous peptides and were run using varying settings to optimize.

\section{Statistical analyses.}

Data were analyzed with a linear model with the design Ratio $\sim$ Genotype, and a one-way analysis of variance using the Tukey test was performed with pairwise contrasts. Contrasts were defined as significant at $P<0.05$. Raw total peak area data were exported from Skyline for analysis and all analyses were run using R (R Core Team 2019) (version 3.6), and the packages nlme (Pinheiro et al. 2021), multcomp (Hothorn et al. 2008), emmeans, multcompview (Graves et al. 2019). Graphs were generated using the package ggplot2 (Wickham 2016).

\section{ACKNOWLEDGMENTS}

The authors thank L. Miller, C. Warwick, E. Kong, M. Bixby, and J. Faust for expert assistance during the mutant screen and laboratory protocols. We also thank D. Kelley for her assistance with Skyline software and peptide selection, M. Taube for sending the raw model of HvSGT1 from their publication, and G. Fuerst for his invaluable assistance throughout the project.

\section{AUTHOR-RECOMMENDED INTERNET RESOURCES}

EZMol v2.1: http://www.sbg.bio.ic.ac.uk/ ezmol/

Proteome Exchange: http://proteomecentral.proteomexchange.org

\section{LITERATURE CITED}

Ade, J., DeYoung, B. J., Golstein, C., and Innes, R. W. 2007. Indirect activation of a plant nucleotide binding site-leucine-rich repeat protein by a bacterial protease. Proc. Natl. Acad. Sci. U.S.A. 104:2531-2536.

Azevedo, C., Betsuyaku, S., Peart, J., Takahashi, A., Noël, L., Sadanandom, A., Casais, C., Parker, J., and Shirasu, K. 2006. Role of SGT1 in resistance protein accumulation in plant immunity. EMBO J. 25:2007-2016.

Azevedo, C., Sadanandom, A., Kitagawa, K., Freialdenhoven, A., Shirasu, K., and Schulze-Lefert, P. 2002. The RAR1 interactor SGT1, an essential component of $R$ gene-triggered disease resistance. Science 295:2073-2076.

Bai, S., Liu, J., Chang, C., Zhang, L., Maekawa, T., Wang, Q., Xiao, W., Liu, Y., Chai, J., Takken, F. L., Schulze-Lefert, P., and Shen, Q. H 2012. Structure-function analysis of barley NLR immune receptor MLA10 reveals its cell compartment specific activity in cell death and disease resistance. PLoS Pathog. 8:e1002752.

Bauer, S., Yu, D., Lawson, A. W., Saur, I. M. L., Frantzeskakis, L., Kracher, B., Logemann, E., Chai, J., Maekawa, T., and Schulze-Lefert, P. 2021. The leucine-rich repeats in allelic barley MLA immune receptors define specificity towards sequence-unrelated powdery mildew avirulence effectors with a predicted common RNase-like fold. PLoS Pathog. 17:e1009223.

Bentele, M., Lavrik, I., Ulrich, M., Stößer, S., Heermann, D. W. Kalthoff, H., Krammer, P. H., and Eils, R. 2004. Mathematical modeling reveals threshold mechanism in CD95-induced apoptosis. J. Cell Biol. 166:839-851.

Bernoux, M., Burdett, H., Williams, S. J., Zhang, X., Chen, C., Newell, K., Lawrence, G. J., Kobe, B., Ellis, J. G., Anderson, P. A., and Dodds, P. N. 2016. Comparative analysis of the flax immune receptors L6 and L7 suggests an equilibrium-based switch activation model. Plant Cell 28:146-159.

Bhattarai, K. K., Li, Q., Liu, Y., Dinesh-Kumar, S. P., and Kaloshian, I. 2007. Mi-1-mediated pest resistance requires $\mathrm{Hsp} 90$ and Sgt1. Plant Physiol. 144:312-323.

Bieri, S., Mauch, S., Shen, Q.-H., Peart, J., Devoto, A., Casais, C., Ceron, F., Schulze, S., Steinbiss, H.-H., Shirasu, K., and Schulze-Lefert, P. 2004. RAR1 positively controls steady state levels of barley MLA resistance proteins and enables sufficient MLA6 accumulation for effective resistance. Plant Cell 16:3480-3495. 
Boter, M., Amigues, B., Peart, J., Breuer, C., Kadota, Y., Casais, C., Moore, G., Kleanthous, C., Ochsenbein, F., Shirasu, K., and Guerois, R. 2007. Structural and functional analysis of SGT1 reveals that its interaction with HSP90 is required for the accumulation of Rx, an R protein involved in plant immunity. Plant Cell 19:3791-3804.

Bourmaud, A., Gallien, S., and Domon, B. 2016. Parallel reaction monitoring using quadrupole-Orbitrap mass spectrometer: Principle and applications. Proteomics 16:2146-2159.

Burke, D., Dawson, D., and Stearns, T. 2000. Methods in Yeast Genetics: A Cold Spring Harbor Laboratory Course Manual, 2000 Edition. Cold Spring Harbor Laboratory Press, Plainview, NY, U.S.A.

Caldo, R. A., Nettleton, D., and Wise, R. P. 2004. Interaction-dependent gene expression in Mla-specified response to barley powdery mildew. Plant Cell 16:2514-2528.

Carneiro, L. S., Martínez, L. C., Gonçalves, W. G., Santana, L. M., and Serrão, J. E. 2020. The fungicide iprodione affects midgut cells of non-target honey bee Apis mellifera workers. Ecotoxicol. Environ. Saf. 189:109991.

Casey, L. W., Lavrencic, P., Bentham, A. R., Cesari, S., Ericsson, D. J., Croll, T., Turk, D., Anderson, P. A., Mark, A. E., Dodds, P. N., Mobli, M., Kobe, B., and Williams, S. J. 2016. The CC domain structure from the wheat stem rust resistance protein Sr33 challenges paradigms for dimerization in plant NLR proteins. Proc. Natl. Acad. Sci. U.S.A. 113:12856-12861.

Chang, C., Yu, D., Jiao, J., Jing, S., Schulze-Lefert, P., and Shen, Q.-H. 2013. Barley MLA immune receptors directly interfere with antagonistically acting transcription factors to initiate disease resistance signaling. Plant Cell 25:1158-1173.

Chapman, A.V.E., Hunt, M., Surana, P., Velásquez-Zapata, V., Xu, W., Fuerst, G., and Wise, R.P. 2020. Disruption of barley immunity to powdery mildew by an in-frame Lys-Leu deletion in the essential protein SGT1. Genetics 217:iyaa026.

Chen, J., Upadhyaya, N. M., Ortiz, D., Sperschneider, J., Li, F., Bouton, C., Breen, S., Dong, C., Xu, B., Zhang, X., Mago, R., Newell, K., Xia, X., Bernoux, M., Taylor, J. M., Steffenson, B., Jin, Y., Zhang, P., Kanyuka, K., Figueroa, M., Ellis, J. G., Park, R. F., and Dodds, P. N. 2017. Loss of AvrSr50 by somatic exchange in stem rust leads to virulence for $\mathrm{Sr} 50$ resistance in wheat. Science 358:1607-1610.

da Silva Correia, J., Miranda, Y., Leonard, N., and Ulevitch, R. 2007. SGT1 is essential for Nod1 activation. Proc. Natl. Acad. Sci. U.S.A. 104:6764-6769.

Draz, I. S., Esmail, S. M., Abou-Zeid, M. A. E.-H., and Essa, T. A E.-M. 2019. Powdery mildew susceptibility of spring wheat cultivars as a major constraint on grain yield. Ann. Agric. Sci. 64:39-45.

Dreze, M., Monachello, D., Lurin, C., Cusick, M. E., Hill, D. E., Vidal, M., and Braun, P. 2010. High-quality binary interactome mapping. Methods Enzymol. 470:281-315.

Fu, D.-Q., Ghabrial, S., and Kachroo, A. 2009. GmRAR1 and GmSGT1 are required for basal, $R$ gene-mediated and systemic acquired resistance in soybean. MPMI 22:86-95.

Gallien, S., Duriez, E., Crone, C., Kellmann, M., Moehring, T., and Domon, B. 2012. Targeted proteomic quantification on quadrupoleorbitrap mass spectrometer. Mol. Cell. Proteomics 11:1709-1723.

Gietz, R. D. 2014. Yeast transformation by the LiAc/SS carrier DNA/ PEG method. Methods Mol. Biol. 1205:1-12.

Godfrey, D., Zhang, Z., Saalbach, G., and Thordal-Christensen, H. 2009. A proteomics study of barley powdery mildew haustoria. Proteomics 9:3222-3232.

Graves, S., Piepho, H.-P., Selzer, L., and Dorai-Raj, S. 2019. multcompView: Visualizations of paired comparisons (R package version 0.1-8). CRAN-R.

Halterman, D. A., and Wise, R. P. 2004. A single-amino acid substitution in the sixth leucine-rich repeat of barley MLA6 and MLA13 alleviates dependence on RAR1 for disease resistance signaling. Plant J. 38:215-226.

Helliwell, C., and Waterhouse, P. 2003. Constructs and methods for high-throughput gene silencing in plants. Methods 30:289-295.

Hothorn, T., Bretz, F., and Westfall, P. 2008. Simultaneous inference in general parametric models. Biomed. J. 50:346-363.

Huber, R. G., Eibl, C., and Fuchs, J. E. 2015. Intrinsic flexibility of NLRP pyrin domains is a key factor in their conformational dynamics, fold stability, and dimerization. Protein Sci. 24:174-181.

Jaswal, R., Kiran, K., Rajarammohan, S., Dubey, H., Singh, P. K., Sharma, Y., Deshmukh, R., Sonah, H., Gupta, N., and Sharma, T. R. 2020. Effector biology of biotrophic plant fungal pathogens: Current advances and future prospects. Microbiol. Res. 241:126567.

Jørgensen, J. H. 1994. Genetics of powdery mildew resistance in barley. Crit. Rev. Plant Sci. 13:97-119.

Joseph, P. R. B., Sawant, K. V., Iwahara, J., Garofalo, R. P., Desai, U. R., and Rajarathnam, K. 2018. Lysines and Arginines play non-redundant roles in mediating chemokine-glycosaminoglycan interactions. Sci. Rep. 8:12289.

Kadota, Y., and Shirasu, K. 2012. The HSP90 complex of plants. Biochim. Biophys. Acta 1823:689-697.

Kadota, Y., Shirasu, K., and Guerois, R. 2010. NLR sensors meet at the SGT1-HSP90 crossroad. Trends Biochem. Sci. 35:199-207.

Klymiuk, V., Coaker, G., Fahima, T., and Pozniak, C. 2021. Tandem protein kinases emerge as new regulators of plant immunity. Mol. PlantMicrobe Interact. 34:1094-1102.

Knebel, C., Heise, T., Zanger, U. M., Lampen, A., Marx-Stoelting, P., and Braeuning, A. 2019. The azole fungicide tebuconazole affects human CYP1A1 and CYP1A2 expression by an aryl hydrocarbon receptor-dependent pathway. Food Chem. Toxicol. 123:481-491.

Krattinger, S. G., and Keller, B. 2016. Molecular genetics and evolution of disease resistance in cereals. New Phytol. 212:320-332.

Kud, J., Zhao, Z., Du, X., Liu, Y., Zhao, Y., and Xiao, F. 2013. SGT1 interacts with the Prf resistance protein and is required for Prf accumulation and Prf-mediated defense signaling. Biochem. Biophys. Res. Commun. 431:501-505.

Kumar, S., and Nussinov, R. 2002. Close-range electrostatic interactions in proteins. ChemBioChem 3:604-617.

Lambertucci, S., Orman, K. M., Das Gupta, S., Fisher, J. P., Gazal, S., Williamson, R. J., Cramer, R., and Bindschedler, L. V. 2019. Analysis of barley leaf epidermis and extrahaustorial proteomes during powdery mildew infection reveals that the PR5 thaumatin-like protein TLP5 is required for susceptibility towards Blumeria graminis f. sp. hordei. Front. Plant Sci. 10:01138.

Lewis, J. D., Wu, R., Guttman, D. S., and Desveaux, D. 2010. Allele-specific virulence attenuation of the Pseudomonas syringae HopZ1a type III effector via the Arabidopsis ZAR1 resistance protein. PLoS Genet. 6:e1000894.

Li, L., Habring, A., Wang, K., and Weigel, D. 2020. Atypical resistance protein RPW8/HR triggers oligomerization of the NLR immune receptor RPP7 and autoimmunity. Cell Host Microbe 27:405-417.e406.

Liu, Z.-q., Liu, Y.-y., Shi, L.-p., Yang, S., Shen, L., Yu, H.-X., Wang, R.-z., Wen, J.-y., Tang, Q., Hussain, A., Khan, M. I., Hu, J., Liu, C.-1., Zhang, Y.-w., Cheng, W., and He, S.-1. 2016. SGT1 is required in PcINF1/SRC2-1 induced pepper defense response by interacting with SRC2-1. Sci. Rep. 6:21651.

Lo Presti, L., Lanver, D., Schweizer, G., Tanaka, S., Liang, L., Tollot, M., Zuccaro, A., Reissmann, S., and Kahmann, R. 2015. Fungal effectors and plant susceptibility. Annu. Rev. Plant Biol. 66:513-545.

Lõoke, M., Kristjuhan, K., and Kristjuhan, A. 2011. Extraction of genomic DNA from yeasts for PCR-based applications. Biotechniques 50:325-328.

Lu, X., Kracher, B., Saur, I. M. L., Bauer, S., Ellwood, S. R., Wise, R., Yaeno, T., Maekawa, T., and Schulze-Lefert, P. 2016. Allelic barley MLA immune receptors recognize sequence-unrelated avirulence effectors of the powdery mildew pathogen. Proc. Natl. Acad. Sci U.S.A. 113:E6486-E6495.

Maekawa, T., Cheng, W., Spiridon, L. N., Töller, A., Lukasik, E., Saijo, Y., Liu, P., Shen, Q. H., Micluta, M. A., Somssich, I. E., Takken, F. L. W., Petrescu, A. J., Chai, J., and Schulze-Lefert, P. 2011. Coiled-coil domain-dependent homodimerization of intracellular barley immune receptors defines a minimal functional module for triggering cell death. Cell Host Microbe 9:187-199.

Maekawa, T., Kracher, B., Vernaldi, S., Ver Loren van Themaat, E., and Schulze-Lefert, P. 2012. Conservation of NLR-triggered immunity across plant lineages. Proc. Natl. Acad. Sci. U.S.A. 109:20119-20123.

Marquenet, E., and Richet, E. 2007. How integration of positive and negative regulatory signals by a STAND signaling protein depends on ATP hydrolysis. Mol. Cell 28:187-199.

Martins, T., Maia, A. F., Steffensen, S., and Sunkel, C. E. 2009. Sgt1, a co-chaperone of Hsp90 stabilizes Polo and is required for centrosome organization. EMBO J. 28:234-247.

Méchin, V., Thévenot, C., Le Guilloux, M., Prioul, J. L., and Damerval, C. 2007. Developmental analysis of maize endosperm proteome suggests a pivotal role for pyruvate orthophosphate dikinase. Plant Physiol. 143:1203-1219.

Meldau, S., Baldwin, I. T., and Wu, J. 2011. For security and stability: SGT1 in plant defense and development. Plant Signal. Behav. 6:1479-1482.

Mestre, P., and Baulcombe, D. C. 2006. Elicitor-mediated oligomerization of the tobacco $\mathrm{N}$ disease resistance protein. Plant Cell 18:491-501.

Mikkelsen, M. D., Buron, L. D., Salomonsen, B., Olsen, C. E., Hansen, B. G., Mortensen, U. H., and Halkier, B. A. 2012. Microbial production of indolylglucosinolate through engineering of a multi-gene pathway in a versatile yeast expression platform. Metab. Eng. 14:104-111.

Noël, L. D., Cagna, G., Stuttmann, J., Wirthmüller, L., Betsuyaku, S., Witte, C.-P., Bhat, R., Pochon, N., Colby, T., and Parker, J. E. 2007. Interaction between SGT1 and cytosolic/nuclear HSC70 chaperones regulates Arabidopsis immune responses. Plant Cell 19:4061-4076. 
Park, C.-J., Caddell, D., and Ronald, P. 2012. Protein phosphorylation in plant immunity: Insights into the regulation of pattern recognition receptor-mediated signaling. Front. Plant Sci. 3:177.

Peart, J. R., Lu, R., Sadanandom, A., Malcuit, I., Moffett, P., Brice, D. C., Schauser, L., Jaggard, D. A. W., Xiao, S., Coleman, M. J., Dow, M., Jones, J. D. G., Shirasu, K., and Baulcombe, D. C. 2002. Ubiquitin ligase-associated protein SGT1 is required for host and nonhost disease resistance in plants. Proc. Natl. Acad. Sci. U.S.A. 99:10865-10869.

Periyannan, S., Moore, J., Ayliffe, M., Bansal, U., Wang, X., Huang, L., Deal, K., Luo, M., Kong, X., Bariana, H., Mago, R., McIntosh, R., Dodds, P., Dvorak, J., and Lagudah, E. 2013. The gene Sr33, an ortholog of barley Mla genes, encodes resistance to wheat stem rust race Ug99. Science 341:786-788.

Peterson, A. C., Russell, J. D., Bailey, D. J., Westphall, M. S., and Coon, J. J. 2012. Parallel reaction monitoring for high resolution and high mass accuracy quantitative, targeted proteomics. Mol. Cell. Proteomics 11:1475-1488.

Pinheiro, J., Bates, D., Debroy, S., Sarkar, D., and Team, R. C. 2021. nlme: Linear and nonlinear mixed effects models ( $\mathrm{R}$ package version 3.1-152). CRAN-R. https://CRAN.R-project.org/package=nlme.

Pino, L. K., Searle, B. C., Bollinger, J. G., Nunn, B., MacLean, B., and MacCoss, M. J. 2020. The Skyline ecosystem: Informatics for quantitative mass spectrometry proteomics. Mass Spectrom. Rev. 39:229-244.

R Core Team. 2019. R: A Language and Environment for Statistical Computing. R Foundation for Statistical Computing, Vienna.

Rauniyar, N. 2015. Parallel reaction monitoring: A targeted experiment performed using high resolution and high mass accuracy mass spectrometry. Int. J. Mol. Sci. 16:28566-28581.

Ridout, C. J., Skamnioti, P., Porritt, O., Sacristan, S., Jones, J. D. G., and Brown, J. K. M. 2006. Multiple avirulence paralogues in cereal powdery mildew fungi may contribute to parasite fitness and defeat of plant resistance. Plant Cell 18:2402-2414.

Saur, I. M. L., Bauer, S., Kracher, B., Lu, X., Franzeskakis, L., Muller, M. C., Sabelleck, B., Kummel, F., Panstruga, R., Maekawa, T., and Schulze-Lefert, P. 2019. Multiple pairs of allelic MLA immune receptor-powdery mildew $\mathrm{AVR}_{\mathrm{A}}$ effectors argue for a direct recognition mechanism. eLife 8:e44471.

Savary, S., Willocquet, L., Pethybridge, S. J., Esker, P., McRoberts, N., and Nelson, A. 2019. The global burden of pathogens and pests on major food crops. Nat. Ecol. Evol. 3:430-439.

Seeholzer, S., Tsuchimatsu, T., Jordan, T., Bieri, S., Pajonk, S., Yang, W., Jahoor, A., Shimizu, K. K., Keller, B., and Schulze-Lefert, P. 2010. Diversity at the Mla powdery mildew resistance locus from cultivated barley reveals sites of positive selection. Mol. Plant-Microbe Interact. 23:497-509.

Shen, Q.-H., Saijo, Y., Mauch, S., Biskup, C., Bieri, S., Keller, B., Seki, H., Ulker, B., Somssich, I. E., and Schulze-Lefert, P. 2007. Nuclear activity of MLA immune receptors links isolate-specific and basal disease-resistance responses. Science 315:1098-1103.

Shirasu, K. 2009. The HSP90-SGT1 chaperone complex for NLR immune sensors. Annu. Rev. Plant Biol. 60:139-164.

Siligardi, G., Zhang, M., and Prodromou, C. 2018. The stoichiometric interaction of the Hsp90-Sgt1-Rar1 complex by CD and SRCD spectroscopy. Front. Mol. Biosci. 4:95.

Sokalingam, S., Raghunathan, G., Soundrarajan, N., and Lee, S.-G. 2012. A study on the effect of surface lysine to arginine mutagenesis on protein stability and structure using green fluorescent protein. PLoS One 7:e40410.

Song, G., Hsu, P. Y., and Walley, J. W. 2018. Assessment and refinement of sample preparation methods for deep and quantitative plant proteome profiling. Proteomics 18:1800220.
Sun, Y., Zhu, Y.-X., Balint-Kurti, P. J., and Wang, G.-F. 2020. Fine-tuning immunity: Players and regulators for plant NLRs. Trends Plant Sci. 25:695-713.

Tameling, W. I. L., Vossen, J. H., Albrecht, M., Lengauer, T., Berden, J. A., Haring, M. A., Cornelissen, B. J. C., and Takken, F. L. W 2006. Mutations in the NB-ARC domain of I-2 that impair ATP hydrolysis cause autoactivation. Plant Physiol. 140:1233-1245.

Taube, M., Pieńkowska, J. R., Jarmołowski, A., Kozak, M., and Silman, I. 2014. Low-resolution structure of the full-length barley (Hordeum vulgare) SGT1 protein in solution, obtained using small-angle X-ray scattering. PLoS One 9:e93313.

Thao, N. P., Chen, L., Nakashima, A., Hara, S.-i., Umemura, K., Takahashi, A., Shirasu, K., Kawasaki, T., and Shimamoto, K. 2007. RAR1 and HSP90 form a complex with Rac/Rop GTPase and function in innate-immune responses in rice. Plant Cell 19:4035-4045.

van Wersch, S., Tian, L., Hoy, R., and Li, X. 2020. Plant NLRs: The whistleblowers of plant immunity. Plant Commun. 1:100016.

Wang, G.-F., Fan, R., Wang, X., Wang, D., and Zhang, X. 2015a. TaRAR1 and TaSGT1 associate with TaHSP90 to function in bread wheat (Triticum aestivum L.) seedling growth and stripe rust resistance. Plant Mol. Biol. 87:577-589.

Wang, G. F., Ji, J., El-Kasmi, F., Dangl, J. L., Johal, G., and Balint-Kurti, P. J. 2015b. Molecular and functional analyses of a maize autoactive NB-LRR protein identify precise structural requirements for activity. PLoS Pathog. 11:e1004674.

Wang, J., Hu, M., Wang, J., Qi, J., Han, Z., Wang, G., Qi, Y., Wang, H.-W., Zhou, J.-M., and Chai, J. 2019a. Reconstitution and structure of a plant NLR resistosome conferring immunity. Science 364:eaav5870.

Wang, J., Wang, J., Hu, M., Wu, S., Qi, J., Wang, G., Han, Z., Qi, Y., Gao, N., Wang, H.-W., Zhou, J.-M., and Chai, J. 2019b. Ligand-triggered allosteric ADP release primes a plant NLR complex. Science 364:eaav5868.

Wei, F., Wing, R. A., and Wise, R. P. 2002. Genome dynamics and evolution of the Mla (powdery mildew) resistance locus in barley. Plant Cell 14:1903-1917.

Wickham, H. 2016. ggplot2: Elegant graphics for data analysis. SpringerVerlag, New York.

Williams, S. J., Sornaraj, P., deCourcy-Ireland, E., Menz, R. I., Kobe, B. Ellis, J. G., Dodds, P. N., and Anderson, P. A. 2011. An autoactive mutant of the $\mathrm{M}$ flax rust resistance protein has a preference for binding ATP, whereas wild-type $\mathrm{M}$ protein binds ADP. Mol. PlantMicrobe Interact. 24:897-906.

Yamaoka, N., Matsumoto, I., and Nishiguchi, M. 2006. The role of primary germ tubes (PGT) in the life cycle of Blumeria graminis: The stopping of PGT elongation is necessary for the triggering of appressorial germ tube (AGT) emergence. Physiol. Mol. Plant Pathol. 69:153-159.

Yang, X., Yang, F., Wang, W., Lin, G., Hu, Z., Han, Z., Qi, Y., Zhang, L., Wang, J., Sui, S.-F., and Chai, J. 2018. Structural basis for specific flagellin recognition by the NLR protein NAIP5. Cell Res. 28:35-47.

Yu, G., Xian, L., Sang, Y., and Macho, A. P. 2019. Cautionary notes on the use of Agrobacterium-mediated transient gene expression upon SGT1 silencing in Nicotiana benthamiana. New Phytol. 222:14-17.

Zhang, J., Coaker, G., Zhou, J.-M., and Dong, X. 2020. Plant immune mechanisms: from reductionistic to holistic points of view. Mol. Plant 13:1358-1378.

Zhang, X., Bernoux, M., Bentham, A. R., Newman, T. E., Ve, T., Casey, L. W., Raaymakers, T. M., Hu, J., Croll, T. I., Schreiber, K. J., Staskawicz, B. J., Anderson, P. A., Sohn, K. H., Williams, S. J., Dodds, P. N., and Kobe, B. 2017. Multiple functional self-association interfaces in plant TIR domains. Proc. Natl. Acad. Sci. U.S.A. 114:E2046-E2052. 